\title{
A New Scanning Method for Fast Atomic Force Microscopy
}

\author{
Iskandar A. Mahmood, S. O. Reza Moheimani, Senior Member, IEEE, and Bharath Bhikkaji
}

\begin{abstract}
In recent years, the atomic force microscope (AFM) has become an important tool in nanotechnology research. It was first conceived to generate 3-D images of conducting as well as nonconducting surfaces with a high degree of accuracy. Presently, it is also being used in applications that involve manipulation of material surfaces at a nanoscale. In this paper, we describe a new scanning method for fast atomic force microscopy. In this technique, the sample is scanned in a spiral pattern instead of the well-established raster pattern. A constant angular velocity spiral scan can be produced by applying single frequency cosine and sine signals with slowly varying amplitudes to the $x$-axis and $y$-axis of AFM nanopositioner, respectively. The use of single-frequency input signals allows the scanner to move at high speeds without exciting the mechanical resonance of the device. Alternatively, the frequency of the sinusoidal set points can be varied to maintain a constant linear velocity (CLV) while a spiral trajectory is being traced. Thus, producing a CLV spiral. These scan methods can be incorporated into most modern AFMs with minimal effort since they can be implemented in software using the existing hardware. Experimental results obtained by implementing the method on a commercial AFM indicate that high-quality images can be generated at scan frequencies well beyond the raster scans.
\end{abstract}

Index Terms-Atomic force microscopy, feedback control, highspeed scanning, spiral scanning, tracking.

\section{INTRODUCTION}

A TOMIC force microscope (AFM) [1], [2] has emerged as a standard tool in nanotechnology research. The working principle of the AFM is based on the use of interactive forces between a tip and a sample surface to sense the proximity of the tip to the sample [3]. The AFM can be used either in contact or noncontact mode. In contact mode, a microcantilever with a very sharp tip is brought in contact with the surface of the sample. In this mode, the repulsive force acting on the tip causes the microcantilever to deflect. The deflection of the cantilever is often measured using a beam-deflection method [2]. In this technique, as illustrated in Fig. 1, a laser beam is reflected at the rear side of the cantilever and the deflection is measured

Manuscript received April 29, 2009; revised November 3, 2009; accepted November 3, 2009. Date of publication November 24, 2009; date of current version March 9, 2011. The review of this paper was arranged by Associate Editor L. Dong.

I. A. Mahmood and S. O. R. Moheimani are with the School of Electrical Engineering and Computer Science, University of Newcastle, Callaghan, N.S.W. 2308, Australia (e-mail: Iskandar.Mahmood@newcastle.edu.au; reza. moheimani@newcastle.edu.au). I. A. Mahmood is on leave from Department of Mechatronics, Faculty of Engineering, International Islamic University Malaysia, 50728, Kuala Lumpur, Malaysia.

B. Bhikkaji is with the Department of Electrical Engineering, Indian Institute of Technology Madras, Chennai 600036, India (e-mail: bharath.bhikkaji@ iitm.ac.in).

Color versions of one or more of the figures in this paper are available online at http://ieeexplore.ieee.org.

Digital Object Identifier 10.1109/TNANO.2009.2036844

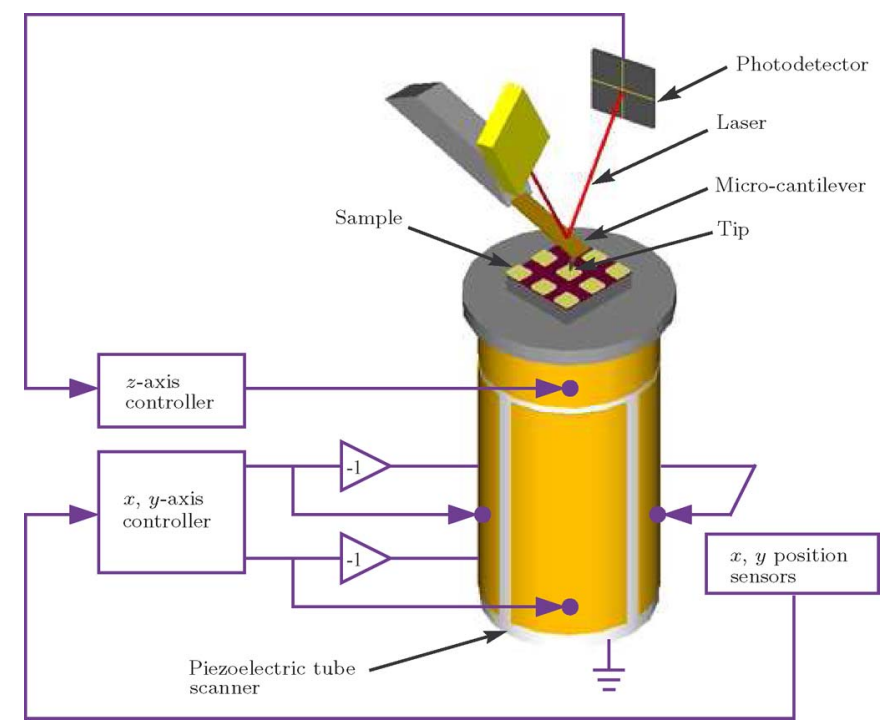

Fig. 1. Basic AFM schematic with feedback controllers.

by a photodetector. In contact mode, the interactive forces are determined by measuring the deflection of the cantilever.

In noncontact mode, the cantilever is made to oscillate. The tip of the cantilever is brought close to, but without coming in contact with, the surface of the sample. AFM images with atomic resolution can be achieved by operating this mode in ultrahigh vacuum (UHV), where the distance between the tip and the sample can be made very small [3]. In this mode, the oscillation of the cantilever is affected by attractive forces present between the tip and the sample surface. In noncontact mode, the interactive forces are measured by measuring changes in oscillation amplitude or resonance frequency of the cantilever. In both modes, the interactive force measurements can be used directly to generate the sample surface topography or as a feedback signal to the $z$-axis controller to keep the interactive force nearly constant.

Today, the majority of commercially available AFMs use raster scans to image a sample's surface. A piezoelectric tube scanner is often used to move the sample or the probe in the $x$, $y$, and $z$ directions [4]. Although, flexure-based nanopositioners [5], [6] have emerged as an alternative to piezoelectric tubes, the latter is still the most widely used nanopositioning technology in AFMs. A raster scan is normally attained by applying a triangular waveform to the fast axis ( $x$-axis) and a staircase, or a very slow ramp signal to the slow axis ( $y$-axis) of the scanner. In order to perform a high-speed raster scan, a high-frequency triangular waveform needs to be used. A triangular waveform contains all odd harmonics of the fundamental frequency. The 
amplitudes of these harmonic signals attenuate as $1 / n^{2}$, with $n$ being the harmonic number [7]. If a fast triangular waveform is applied to the scanner, it will inevitably excite the mechanical resonance of the device. Consequently, this causes the scanner to vibrate and trace a distorted triangular waveform along the $x$ axis, which can significantly distort the generated AFM image. To avoid this complication, the scanning speed of AFMs is often limited to about 10-100 times lower then the scanner's first resonance frequency. Apart from these vibrations, the accuracy of the AFM images are also affected by a number of nonlinear properties that are inherent to piezoelectric material, including hysteresis, creep, and drift [8], [9].

A widely used approach to deal with these issues is to use feedback-control techniques to track the triangular waveform. One of the earliest attempts is reported in [10], where Lag-lead and $H_{\infty}$ controllers were designed and implemented on the piezoelectric tube scanner, and the feasibility of reducing the adverse effects of creep and hysteresis were demonstrated. They also reported that the $H_{\infty}$ controller achieved damping of high-frequency vibrations. In [11], loop-shaping techniques were used to design a feedback controller for a piezoelectric tube scanner to track a raster pattern. The closed-loop system was implemented on an AFM and their results show that accurate positioning with a high degree of repeatability is achievable with the use of feedback control. In [12], a proportional-plus-derivative (PD) high-gain feedback controller and a feedforward input were used to compensate for creep, hysteresis, and vibration effects in an AFM piezoactuator system. The high-gain feedback controller was first used to linearize the piezoactuator by compensating for the creep and hysteresis. Then, the linearized piezoactuator was modeled to determine the feedforward input to account for the vibration effects. Their results indicated that the inclusion of feedforward input reduces the tracking error more as compared to using only feedback control. Other examples of successful applications of feedback-control techniques include [13]-[17]. An exhaustive review of the literature can be found in [9].

The use of feedback controllers in damping and linearizing the piezoelectric tube scanner has been shown to be successful in the aforementioned works [10]-[17]. However, these feedback controllers have little success in tracking high-frequency triangular waveforms. Closed-loop tracking of these waveforms typically results in the corners of the triangular waveform to be rounded off. This is due to the presence of high-frequency harmonics that are inevitably outside of the bandwidth of the closed-loop system. Consequently, AFM images generated at high speeds often demonstrate significant distortions especially around the edges of the image.

This paper proposes a new scan technique for fast atomic force microscopy by forcing the scanner to follow a spiral trajectory over the surface that is to be imaged. A constant angular velocity (CAV) spiral scan can be produced by applying slowly varyingamplitude single-frequency sinusoidal signals to the $x$ and $y$ axes of the piezoelectric tube scanner. The use of the singlefrequency input signals allows for scanning to be performed at very high speeds without exciting the resonance of the scanner and with relatively small control efforts. An alternative method is to generate the spiral pattern in a constant linear velocity (CLV) approach. The latter method has been implemented in some disk storage devices, such as CD-ROM, where the information is stored in a continuous spiral track over the disk's surface [18]. The proposed method is an alternative to rasterbased sinusoidal scan methods that are used to achieve fast scans in, e.g., scanning near-field optical microscopy (SNOM) [19]. In spiral scanning, both axes follow sinusoidal signals of identical frequencies, resulting in a smooth trajectory. This avoids the transient behavior that may occur in sinusoidal scans as the probe moves from one line to the next. Furthermore, the proposed method does not require specialized hardware, e.g., a tuning fork actuator, and can be implemented on a standard AFM with minor software modifications.

It should be noted that recently a few prototype laboratory AFMs have been developed that are capable of imaging a sample at, or close to, video rates, [20]-[23]. Such a functionality is particularly useful in a number of applications, e.g., when capturing the dynamic behavior of certain biomolecular processes is needed. In order to achieve video rates, using a raster-scanned AFM, the scan frequency has to be quite high, close to $5 \mathrm{kHz}$ or higher. The spiral scanning method proposed in this paper may be a good candidate for such applications. This scheme can be easily implemented on a commercial, or prototype AFM, with minimal software modifications. It should be pointed out that to operate an AFM at such high scan frequencies, one has to overcome other technical challenges, such as the need to utilize very small microcantilevers with extremely high resonance frequencies [20].

The remainder of this paper is organized as follows. The generation of input signals to produce the spiral pattern is described in detail in Section II. Section III provides descriptions of the AFM and other experimental setups used in this paper. Modeling and identification of the system transfer functions are presented in Section IV. Control schemes for the AFM scanner are devised in Section V. In Section VI, experimental results are presented to illustrate the drastic improvement in imaging speed that can be achieved with the proposed new scan trajectory. Finally, Section VII concludes the paper.

\section{SPIRAL SCAN}

This section deals with the generation of input signals that are needed to move the AFM scanner in a spiral pattern, as illustrated in Fig. 2. The pattern is known as the Archimedean spiral. A property of this spiral is that its pitch $P$, which is distance between two consecutive intersections of the spiral curve with any line passing through the origin, is constant [24]. Depending on how this trajectory is traced, the shape is referred to as either a CAV spiral, or a CLV spiral. In the former case, the pattern is traced at a CAV, while in the latter at a CLV.

\section{A. CAV Spiral}

The equation that generates a CAV spiral of pitch $P$ at an angular velocity of $\omega$ can be derived from a differential equation 


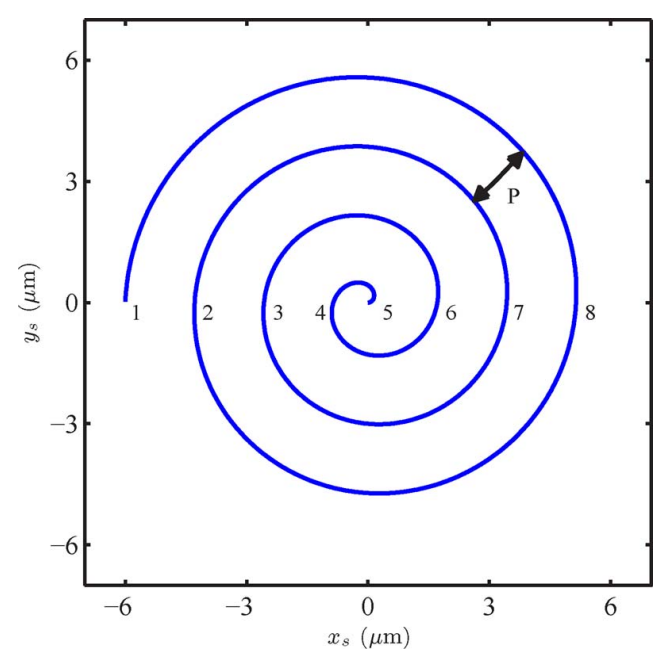

Fig. 2. Spiral scan of 6- $\mu \mathrm{m}$ radius with number of curve $=8$.

given in [18] as

$$
\frac{d r}{d t}=\frac{P \omega}{2 \pi}
$$

where $r$ is the instantaneous radius at time $t$. Equation (1) is solved for $r$ by integrating both sides to obtain

$$
\int d r=\frac{P \omega}{2 \pi} \int d t
$$

For $r=0$ at $t=0$, we have

$$
r=\frac{P}{2 \pi} \omega t
$$

Here, $P$ is calculated as

$$
P=\frac{\text { spiral radius } \times 2}{\text { number of curves }-1}
$$

where number of curves is defined as the number of times the spiral curve crosses through the line $y=0$. This is exemplified in Fig. 2, where the crossing points are numbered. The figure illustrates a spiral scan of $6 \mu \mathrm{m}$ radius with number of curves $=8$.

The equation that describes the total scanning time $t_{\text {total }}$ associated with a CAV spiral scan can be derived by integrating both sides of (1) as

$$
\int_{r_{\mathrm{start}}}^{r_{\mathrm{end}}} d r=\frac{P \omega}{2 \pi} \int_{t_{\mathrm{start}}}^{t_{\mathrm{end}}} d t
$$

where $r_{\text {start }}$ and $r_{\text {end }}$ are initial and final values of the spiral radius, and $t_{\text {start }}$ and $t_{\text {end }}$ are initial and final values of the scanning time. From (5), if $r_{\text {start }}=0$ at $t_{\text {start }}=0$ and $t_{\text {total }}=$ $t_{\text {end }}-t_{\text {start }}$, we obtain

$$
t_{\text {total }}=\frac{2 \pi r_{\text {end }}}{P \omega} .
$$

In order to implement the spiral scans using a piezoelectric tube scanner, (3) needs to be translated into Cartesian coordinates. The transformed equations are as follows:

$$
x_{s}=r \cos \theta
$$

and

$$
y_{s}=r \sin \theta
$$

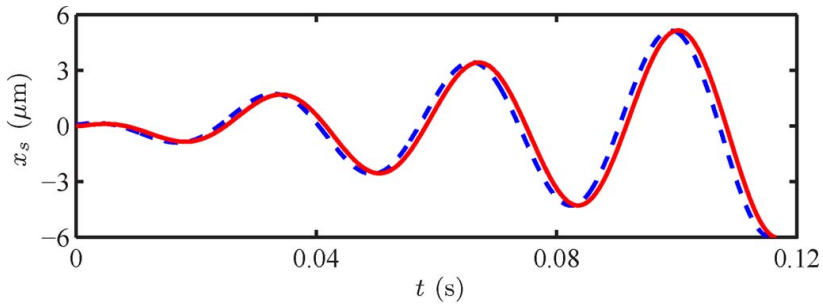

(a)

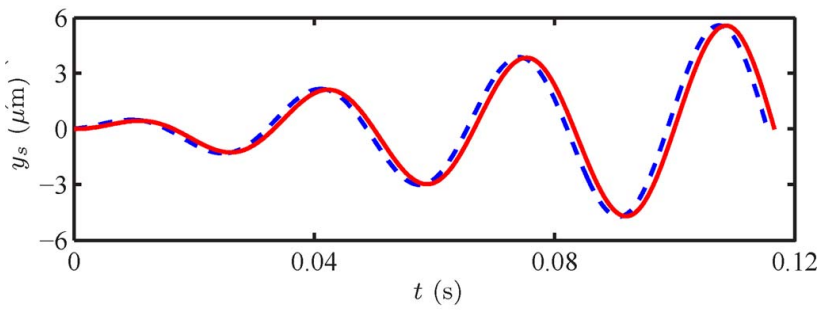

(b)

Fig. 3. Input signals to be applied to the scanner in the $x$ and $y$ axes of the scanner to generate CAV spiral scan with $\omega=188.50 \mathrm{rad} / \mathrm{s}$. Solid line is the achieved response and dashed line is the desired trajectory.

where $x_{s}$ and $y_{s}$ are input signals to be applied to the scanner in the $x$ and $y$ axes, respectively and $\theta$ is the angle. From $\omega=$ $d \theta / d t, \theta$ is obtained as $\theta=\omega t$. An example of input signals $x_{s}$ and $y_{s}$ that can generate the spiral in Fig. 2 is plotted in Fig. 3. The figure illustrates constant phase errors between the input signals and measured outputs. Such errors are due to the nonideal frequency response of the controlled nanopositioner. For a CAV spiral, these phase errors can be easily eliminated by adding phase constants $\alpha_{x}$ and $\alpha_{y}$ to shape the input signals as

$$
X_{s}=r \cos \left(\theta+\alpha_{x}\right)
$$

and

$$
Y_{s}=r \sin \left(\theta+\alpha_{y}\right) .
$$

Here, $\alpha_{x}$ and $\alpha_{y}$ are determined by measuring the closed-loop frequency response of the system at the scan frequency. They may also be determined off-line if a model of the system is at hand.

A key advantage of using a CAV spiral is that closed-loop tracking of this pattern, when implemented via the Cartesian equations, only involves tracking single-frequency sinusoidal signals with slowly varying amplitudes. This advantage, when combined with the use of the shaped input signals (9) and (10), enables the AFM's scanner to track a high-frequency CAV spiral resulting in fast atomic force microscopy. A drawback of this method is that its linear velocity $v$ is not constant. Thus, it may not be suitable for scanning some samples, where the interaction between the probe and the sample needs to be done at linear velocity. The CLV spiral presented next overcomes this problem.

\section{B. CLV Spiral}

In order to generate a CLV spiral, the radius $\widetilde{r}$ and angular velocity $\widetilde{\omega}$ need to be varied simultaneously in a way that the linear velocity of the nanopositioner is kept constant at all times. The expressions for $\widetilde{r}$ and $\widetilde{\omega}$ are first derived by substituting 
$\omega=v / r$ into (1) to obtain

$$
\frac{d r}{d t}=\frac{P v}{2 \pi r}
$$

where $v$ is the linear velocity of the CLV spiral. Then, (11) is solved for $r$ by integrating both sides of the equation as

$$
\int r d r=\frac{P v}{2 \pi} \int d t
$$

For $r=0$ at $t=0$, we obtain

$$
\widetilde{r}=\sqrt{\frac{P v}{\pi} t} .
$$

From (13), by substituting $\widetilde{r}=v / \widetilde{\omega}$, the expression for $\widetilde{\omega}$ is obtained as

$$
\widetilde{\omega}=\sqrt{\frac{\pi v}{P t}} .
$$

It is worth noting that $\widetilde{r}$ and $\widetilde{\omega}$ are nonlinear functions of time, and $\widetilde{\omega}$ approaches infinity at $t=0$. For practical reasons during digital implementation of the CLV spiral, $t=0$ is approximated with $t=$ sampling period of the digital system.

The equation for total scanning time $\widetilde{t}_{\text {total }}$ of a CLV spiral scan can be derived in a similar manner to the CAV spiral. From (11), $\widetilde{t}_{\text {total }}$ is derived as follows:

$$
\tilde{t}_{\text {total }}=\frac{\pi r_{\mathrm{end}}^{2}}{P v} .
$$

By choosing $v=\widetilde{\omega}_{\text {end }} r_{\text {end }}$, where $\widetilde{\omega}_{\text {end }}$ is the instantaneous angular velocity at $r_{\text {end }}$, the equation for $\widetilde{t}_{\text {total }}$ can be rewritten as

$$
\widetilde{t}_{\text {total }}=\frac{\pi r_{\text {end }}}{P \widetilde{\omega}_{\text {end }}} \text {. }
$$

It can be inferred from (16) that if $\widetilde{\omega}_{\text {end }}=\omega$, the total scanning time of a CLV spiral is half of the total scanning time of a CAV spiral. This makes the CLV spiral a more attractive option. However, as we will see later, this gain in scanning time comes at the expense of introducing distortion at the center of the AFM image.

Similar to the CAV spiral, (13) can be described in Cartesian coordinates as

$$
\widetilde{x}_{s}=\widetilde{r} \cos \widetilde{\theta}
$$

and

$$
\widetilde{y}_{s}=\widetilde{r} \sin \theta
$$

where $\widetilde{\theta}$ for time-varying $\widetilde{\omega}$ is obtained as follows:

$$
\widetilde{\theta}=\sqrt{\frac{4 \pi v}{P} t} .
$$

Fig. 4 illustrates the input signals $\widetilde{x}_{s}$ and $\widetilde{y}_{s}$ that can be used to generate a spiral similar to the one shown in Fig. 2. However, as illustrated in the figure, the input signals are implemented in a reversed order, which is from $r_{\text {end }}$ to $r_{\text {start }}$. To generate a CLV spiral, which starts from $\widetilde{r}=0$, one requires a closed-loop system with extremely high bandwidth (ideally $\infty$ bandwidth) and a closed-loop system with a flat phase and magnitude response. This of course, is not practical. Thus, if the spiral is

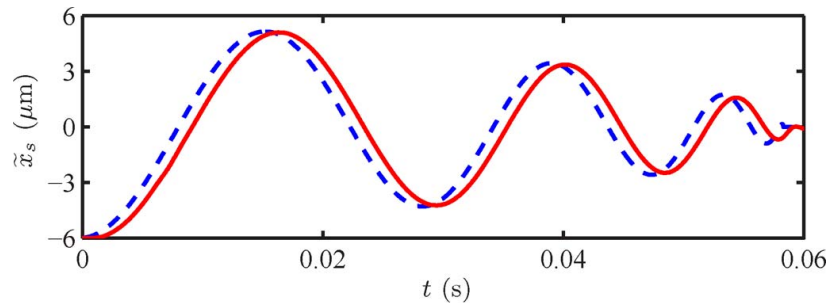

(a)

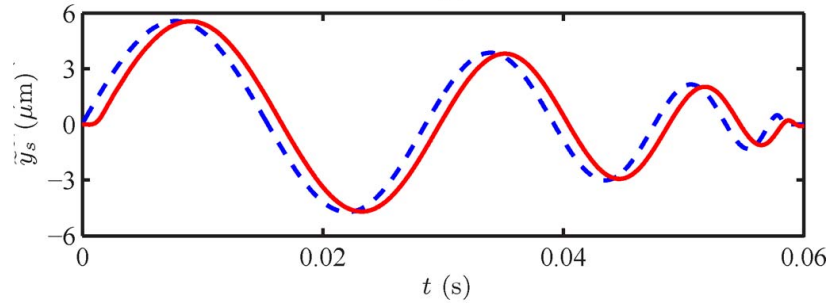

(b)

Fig. 4. Input signals to be applied to the scanner in the $x$ and $y$ axes of the scanner to generate CLV spiral scan with $v=1.13 \mathrm{~mm} / \mathrm{sec}$ (or $\widetilde{\omega}_{\text {end }}=$ $188.50 \mathrm{rad} / \mathrm{s}$ ). Solid line is the achieved response and dashed line is the desired trajectory.

started from $\widetilde{r}=0$, the initial error that is inevitably generated, will propagate all the way through to the end. In the next section, we propose an inversion algorithm that can minimize the tracking error arising from the limited bandwidth and nonideal frequency response of the closed-loop system.

\section{Inversion Technique for CLV Spiral}

In this section, a technique to shape inputs such that the resulting trajectory will be a CLV spiral with minimal tracking error is presented. As the implementation of the entire scheme will be in discrete time, the input-shaping method, presented here, is also described in discrete time.

The goal is to design input signals $\left\{u_{x}[k]\right\}_{k=0}^{N}$ and $\left\{u_{y}[k]\right\}_{k=0}^{N}$, such that their outputs, along the $x$ and $y$ axes are $\{x[k]=\tilde{x}(k T)\}_{k=0}^{N}$ and $\{y[k]=\tilde{y}(k T)\}_{k=0}^{N}$, respectively. Here, $T$ denotes the sampling interval and $\tilde{x}$ and $\tilde{y}$ are as defined in (17) and (18). In the following, only designing of $\left\{u_{x}[k]\right\}_{k=0}^{N}$ will be described, with the understanding that $\left\{u_{y}[k]\right\}_{k=0}^{N}$ can be generated by adopting the same procedure.

Assume that the transfer function relating the input and the output along the $x$-direction is given by

$$
G_{x}(z)=\frac{b_{0}+b_{1} z^{-1}+b_{2} z^{-2}+\cdots+b_{m} z^{-m}}{1+a_{1} z^{-1}+a_{2} z^{-2}+\cdots+a_{m} z^{-m}}
$$

which is stable, but has nonminimum phase zeros, i.e., all of zeros are outside the unit circle. As $G_{x}(z)$ is nonminimum phase, a direct inversion is not possible. Furthermore, as $\tilde{x}$ and $\tilde{y}$ are not periodic, a frequency-domain inversion of the type presented in [15] will not be accurate.

Note that (20) in the discrete time corresponds to the difference equation as

$$
\begin{aligned}
x[n]+ & a_{1} x[n-1]+\cdots+a_{m} x[n-m] \\
& =b_{0} u_{x}[n]+b_{1} u_{x}[n-1]+\cdots+b_{m} u_{x}[n-m] .
\end{aligned}
$$


This implies

$$
\begin{aligned}
u_{x}[n-m]= & \frac{1}{b_{m}}\left(x[n]+a_{1} x[n-1]+\cdots+a_{m} x[n-m]\right. \\
& \left.-b_{n} u_{x}[n]-\cdots-b_{m-1} u_{x}[n-(m-1)]\right) .
\end{aligned}
$$

As $\{x[k]\}_{k=0}^{N}$ is given, assuming arbitrary values for $u_{x}[N]$, $u_{x}[N-1], \ldots, u_{x}[N-(m-1)]$, the input sequence $u_{x}[N-$ $(m-1)], u_{x}[N-(m-2)], \ldots, u_{x}[1], u_{x}[0]$ can be calculated from (22). As an example, consider $m=2$ in (21). This implies

$$
\begin{aligned}
& x[n]+a_{1} x[n-1]+a_{2} x[n-2] \\
& \quad=b_{0} u_{x}[n]+b_{1} u_{x}[n-1]+b_{2} u_{x}[n-2]
\end{aligned}
$$

and

$$
\begin{aligned}
u_{x}[n-2]= & \frac{1}{b_{2}}\left(x[n]+a_{1} x[n-1]\right. \\
& \left.+a_{2} x[n-2]-b_{0} u_{x}[n]-b_{1} u_{x}[n-1]\right) .
\end{aligned}
$$

Setting $u_{x}[N]$ and $u_{x}[N-1]$ to arbitrary values, $u_{x}[N-2]$ can be back-calculated from (24). Similarly, using the calculated $u_{x}[N-2]$ and the arbitrarily chosen $u_{x}[N-1], u_{x}[N-3]$ can be computed. Thus, traversing backward in time, one can compute $u_{x}[n]$ up to $n=0$.

The aforementioned procedure can be proved to be stable, and can be shown to converge to an input sequence that would generate the output $\tilde{x}(k T)$. However, the proof is beyond the scope of this paper. If a user has to deal with a continuous time transfer function $G_{x}(s)$, he or she could approximate it by a discrete transfer function $G_{x}(z)$ using the bilinear transformation or any other approximation technique.

\section{Total Scan Time: Spiral Scan Versus Raster Scan}

A fair comparison of the total scanning time for spiral and raster scans can be made by evaluating the time required for both methods to generate images of equal areas and pitch lengths. The area of a circular spiral scanned image $A_{\text {spiral }}$ with a radius of $r_{\text {end }}$ can be calculated as

$$
A_{\text {spiral }}=\pi r_{\text {end }}^{2} .
$$

The area of a rectangular raster-scanned image $A_{\text {raster }}$ can be calculated using

$$
A_{\text {raster }}=L^{2}
$$

where $L$ is length of the square image. For both images to have an equal area, (25) and (26) are equated to obtain

$$
L=\sqrt{\pi} r_{\text {end }} .
$$

The number of lines in a raster-scanned image with pitch $P$ can be calculated as

$$
\text { number of lines }=\frac{L}{P}+1 \text {. }
$$

The total scan time to generate a raster-scanned image can be obtained using

$$
t_{\text {total raster }}=\frac{\text { number of lines }}{f}
$$

where $f$ is the scan frequency. Thus, by substituting (27) and (28) into (29), the total scan time for generating a raster-scanned image with an area of $\pi r_{\text {end }}^{2}$ can be determined as

$$
t_{\text {total raster }}=\frac{\sqrt{\pi} r_{\text {end }}}{P f}+\frac{1}{f} .
$$

The total scanning time to generate a spiral scanned image in a CAV mode can be calculated using (6) and by substituting $\omega=2 \pi f$ into (6)

$$
t_{\mathrm{total}}=\frac{r_{\mathrm{end}}}{P f} .
$$

It can be deduced from (30) and (31), by ignoring the term $1 / f$ in (30), for the same scan frequency, an image of equal area and pitch can be generated $\sqrt{\pi}(\approx 1.77)$ times faster using a CAV spiral scan than a raster scan.

In order to compare the total scanning time for a CLV spiral scan and a raster scan, the linear velocity of the raster scan $v_{r}=2 L f$ is introduced into (30) to obtained

$$
t_{\text {total raster }}=\frac{2 \pi r_{\text {end }}^{2}}{P v_{r}}
$$

with the term $1 / f$ ignored. It can be deduced from (32) and (15), for the same linear velocity, $v_{r}=v$, an image of equal area and pitch can be generated two times faster using a CLV spiral scan than a raster scan.

\section{E. Total Trajectory Distance: Spiral Scan Versus Raster Scan}

In this section, total trajectory distance in generating images of equal area and pitch length using spiral and raster scans are compared. The total trajectory distance of a spiral scan $s_{\text {spiral }}$ can be derived from (15) as follows:

$$
s_{\text {spiral }}=\frac{\pi r_{\text {end }}^{2}}{P} .
$$

Note that (33) also holds for a CAV spiral scan.

The total trajectory of a raster scan $s_{\text {raster }}$ can be written as follows:

$$
s_{\text {raster }}=2 L \times \text { number of lines. }
$$

By substituting (28) and (27) into (34), $s_{\text {raster }}$ can be obtained as follows:

$$
s_{\text {raster }}=\frac{2 \pi r_{\text {end }}^{2}}{P}+2 \sqrt{\pi} r_{\text {end }} .
$$

It can be deduced from (35) and (33) that $s_{\text {raster }}$ is at least two times longer than $s_{\text {spiral }}$. An immediate advantage of this is that there will be less wear on the AFM tip when the spiral scan is used instead of the raster scan.

\section{F. Mapping Spiral Points to Raster Points}

In this paper, the spiral-scanned images are plotted by mapping the sampling points along the spiral trajectory (called "spiral points") to points or pixels (called "raster points") that make up a raster-scanned image placed on top of the spiral points, as shown in Fig. 5(a) and (b) for CAV and CLV spirals, respectively. A major advantage of mapping the spiral points to the 


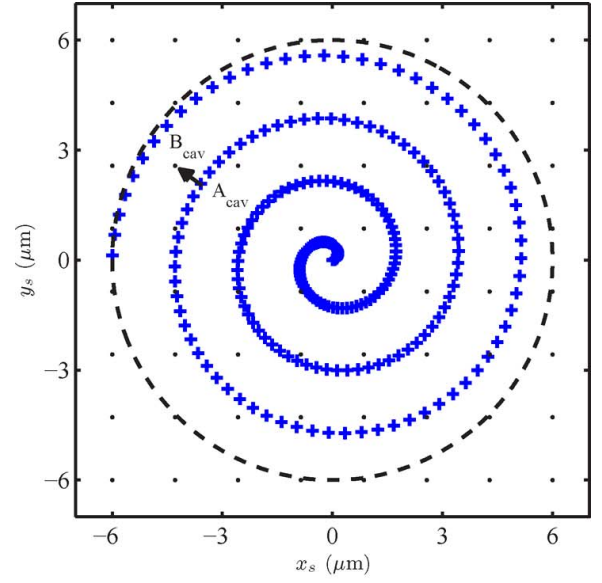

(a)

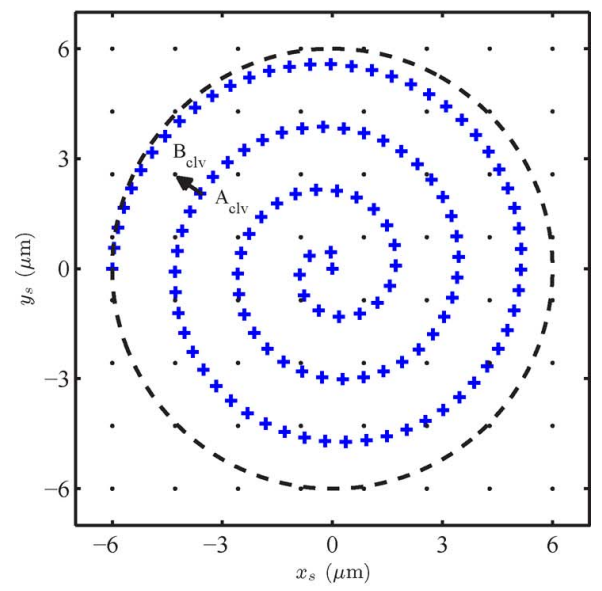

(b)

Fig. 5. Spiral points (+) for (a) CAV spiral with $\omega_{s}=188.5 \mathrm{rad} / \mathrm{s}$ and (b) CLV spiral with $v_{s}=1.1 \mathrm{~mm} / \mathrm{s}$. The sampling frequency used for generating these spiral points is $2 \mathrm{kHz}$. Both spiral trajectories have a $6.5-\mu \mathrm{m}$ radius with number of curves $=8$. The spiral points are plotted on top of the raster points (.) that make up a $13 \times 13 \mu \mathrm{m}$ raster-scanned image with of $8 \times 8$ pixels resolution.

raster points is that it allows the user to utilize existing imageprocessing software developed specifically for raster-scanned images, to plot the generated spiral image.

In this mapping procedure, the dimension of the rasterscanned image is set to spiral diameter $\times$ spiral diameter where the spiral diameter $\approx$ spiral radius $\times 2$, and the pitch of the raster-scanned image is chosen to be equal to the pitch $P$ of the spiral. Consequently, the number of lines in the raster-scanned image will be equal to the number of curves in the spiral trajectory. Then, each raster point located within the spiral radius is mapped to the nearest spiral point. Since position of the raster and spiral points are known for any scan frequency and dimension, the nearest spiral point corresponding to each raster point can be identified and stored in an indexed matrix before performing the sample scans. By doing this, the image of the sample can be plotted in real time, i.e., as the AFM is scanning the sample.

Fig. 5(a) illustrates that the density of the CAV spiral points increases as $r$ approaches the origin of the spiral. This is because

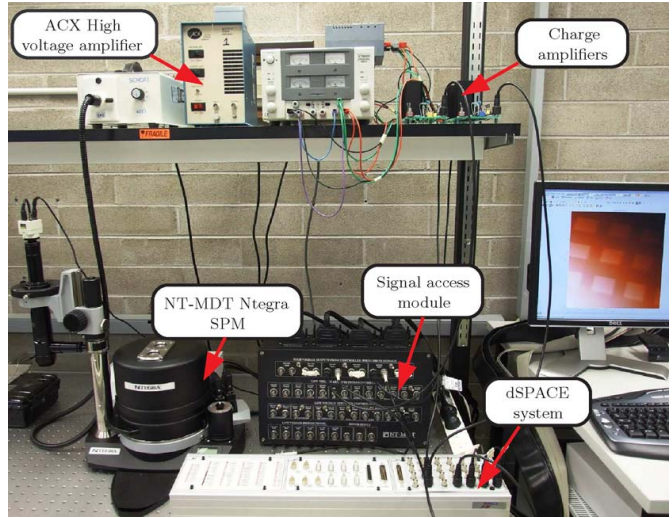

Fig. 6. SPM system and experimental setup used in this paper.

the time taken for the spiral trajectory to make one full spiral circle remains constant due to the CAV, although the circumference of the spiral circle gets smaller. A disadvantage of this is that it increases the computing time needed to search for the nearest spiral point corresponding to each raster point. Nevertheless, Fig. 5(b) shows that the density of the CLV spiral points remain constant for the entire spiral trajectory. This is because in the CLV spiral, the time taken for the spiral trajectory to make one full circle reduces as $r$ approaches the origin of the spiral.

Next, the error introduced by mapping the spiral points to the raster points is analyzed. This mapping error can be determined by calculating the magnitude of the vector between the nearest spiral point corresponding to each raster point. Fig. 5(a) illustrates an example of the vector between spiral point $A_{\mathrm{CAV}}$ and raster point $B_{\mathrm{CAV}}$ which corresponds to raster point $(2,6)$. The magnitude of this vector can be calculated as follows:

$$
\left|A B_{\mathrm{CAV}}^{(i, j)}\right|=\sqrt{\left(A_{\mathrm{CAV}}^{x}-B_{\mathrm{CAV}}^{x}\right)^{2}+\left(A_{\mathrm{CAV}}^{y}-B_{\mathrm{CAV}}^{y}\right)^{2}}
$$

where $i=2$ and $j=6$. Similar calculation can be performed on the CLV spiral to evaluate the magnitude of the vector between spiral point $A_{\mathrm{CLV}}$ and raster point $B_{\mathrm{CLV}}$, as shown in Fig. 5(b).

\section{SYSTEM DESCRIPTION}

The experimental setup consisted of a commercial NT-MDT Ntegra scanning probe microscope (SPM), as shown in Fig. 6. The SPM can be used to perform almost all scanning probe microscopy techniques in air and liquid environment. The SPM's operating software limits the highest image resolution to $256 \times$ 256 pixels. At this resolution, the fastest scanning frequency is limited to $31.25 \mathrm{~Hz}$. However, faster scan frequencies are possible by reducing the resolution. For example, by halving the resolution to $128 \times 128$ pixels, the fastest scan frequency is doubled to $62.50 \mathrm{~Hz}$.

In the experiments reported in this paper, the SPM was configured to operate as an AFM and all sample images were scanned in air. The voltage amplifiers that drive the lateral axes of the piezoelectric tube scanner were replaced with two home-made dc-accurate charge amplifiers [25]. The charge amplifiers have a constant gain of $68 \mathrm{nC} / \mathrm{V}$. The use of the charge amplifiers to drive the piezoelectric tube has been shown to result in a 


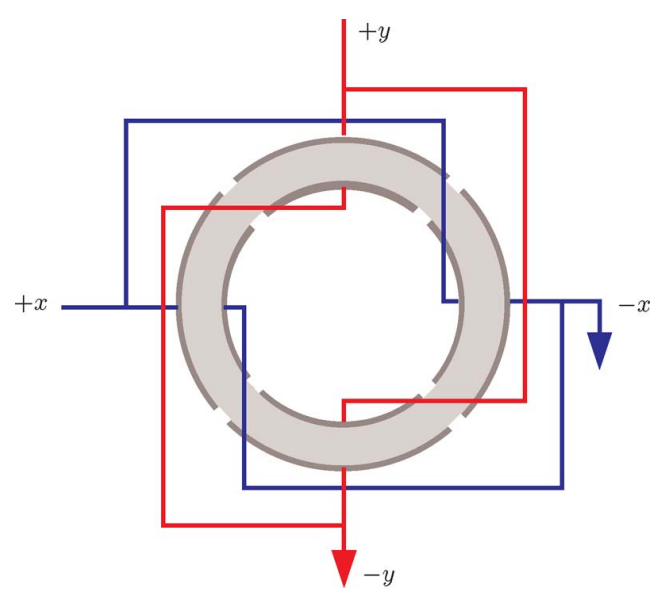

Fig. 7. Top view of the piezoelectric tube with the internal and external electrode wired in a bridge configuration.

reduction of the hysteresis by greater than $90 \%$, as compared with when voltage amplifiers are used [26]. A dSPACE-1103 rapid prototyping system was used to implement the feedback controllers in real time. The amplifiers and the SPM were interfaced with the dSPACE system using a signal access module (SAM) that allowed direct access to the scanner electrodes. This setup enabled us to directly control the lateral movements of the scanner. However, the scanner vertical positioning was achieved using the standard NT-MDT SPM controller.

There are two types of scanner that can be incorporated into the SPM, an open-loop scanner (without displacement sensors) and a closed-loop scanner (with displacement sensors). The closed-loop scanner allows accurate position tracking through feedback control. In these experiments, a closed-loop scanner NT-MDT Z50309cl was used to perform 3-D positioning in the SPM. It has a scanning range of $100 \times 100 \times 10 \mu \mathrm{m}$. The capacitive sensors that are incorporated into the scanner apparatus allow for direct measurements of the scanner displacement in $x, y$, and $z$ axes. The bandwidth of these capacitive sensors is tunable and has a maximum value of $10 \mathrm{kHz}$. In these experiments, the bandwidth is set to the maximum in order to minimize the effect of the capacitive sensors dynamics on the displacement measurements. The sensitivity of the capacitive sensors was determined by making the scanner to track a $0.5-\mathrm{Hz}$ triangular wave of $100-\mu \mathrm{m}$ amplitude in closed loop using the standard NT-MDT SPM controller. Simultaneously, the corresponding output voltages from the capacitive sensors were also measured. From these two values, the sensitivity of the capacitive sensors incorporated into the $x$ and $y$ axes was calculated to be $6.33 \mu \mathrm{m} /$ volt. In this test, a low-frequency triangular wave was used to ensure perfect tracking by the standard NT-MDT SPM controller.

The piezoelectric tube in the scanner has quartered internal and external electrodes. Such electrode arrangement allows the scanner to be driven in a bridge configuration [26], where the electrodes are wired in pairs, as illustrated in Fig. 7. These electrode pairs are referred to as $+x,-x,+y$, and $-y$ electrode pairs. An advantage of using the bridge configuration is that it halves the input voltage requirement, as compared to the more

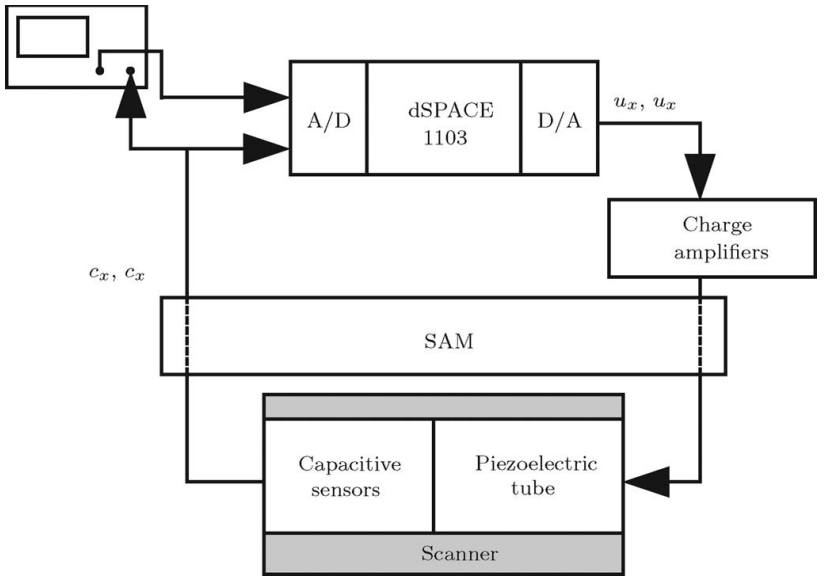

Fig. 8. Block diagram of the experimental setup used for system identification of the scanner.

widely used grounded internal electrode configuration. Nevertheless, in these experiments, the $-x$ and $-y$ electrodes are grounded in order to simplify the experimental setup. Furthermore, the need for a large scanning range does not arise here since the scanner is only made to operate within $12 \%$ of its full lateral scanning range.

During scans, measurements from the capacitive sensors and photodiode are recorded and processed in MATLAB to generate AFM images.

\section{SySTEM IDENTIFICATION}

In this section, the procedure used to model the AFM scanner is described. The scanner is treated as a two single-input singleoutput (SISO) systems in parallel. The inputs being the voltage signals applied to the charge amplifiers driving $+x$ electrode pair $u_{x}$ and $+y$ electrode pair $u_{y}$. The outputs of the system are the scanner displacement measurements from the capacitive sensors in $x$-axis $c_{x}$ and in $y$-axis $c_{y}$. Here, accurate models of the systems were obtained through system identification. System identification is an experimental approach to modeling, where mathematical models are obtained from a set of input and output data [27].

Fig. 8 illustrates the experimental setup used for the system identification experiment. A dual-channel HP35670A spectrum analyzer was used to obtain the following frequency response functions (FRFs) nonparametrically:

$$
G_{c_{x} u_{x}}(i \omega)=\frac{c_{x}(i \omega)}{u_{x}(i \omega)}
$$

and

$$
G_{c_{y} u_{y}}(i \omega)=\frac{c_{y}(i \omega)}{u_{y}(i \omega)}
$$

A band-limited random noise signal of amplitude $0.5 \mathrm{Vpk}$ within the frequency range of $1-1600 \mathrm{~Hz}$ was generated using the spectrum analyzer and applied to the charge amplifiers as the input. The corresponding outputs from the capacitive displacement sensors were also recorded using the spectrum analyzer. 

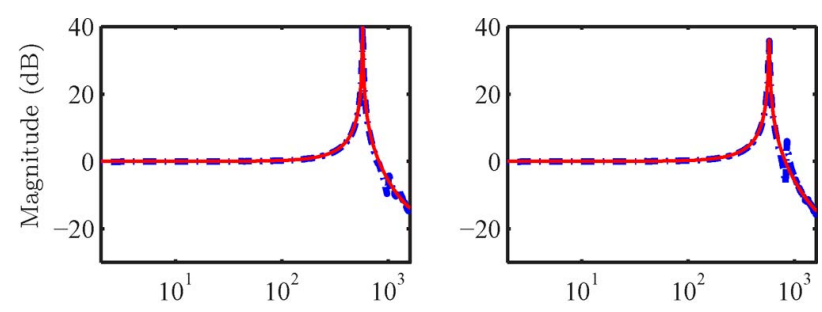

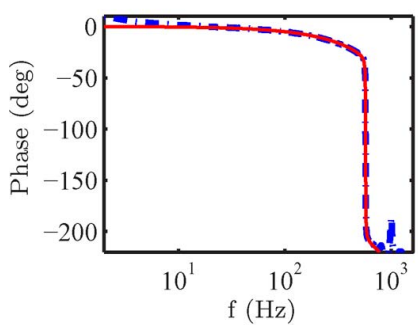

(a)

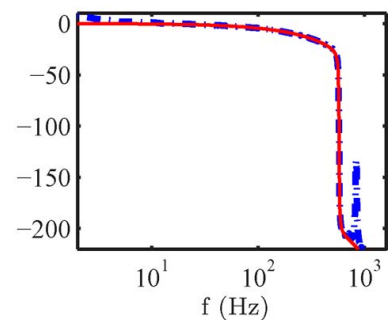

(b)
Fig. 9. Experimental (--) and identified model (-) frequency response of (a) $G_{c_{x} u_{x}}(s)$ and (b) $G_{c_{y} u_{y}}(s)$.

These input-output data were processed to generate the FRF (37) and (38) in a nonparametric form, as illustrated in Fig. 9. Note that the $0 \mathrm{~dB}$ (unity gain) at dc in both FRFs was achieved by introducing appropriate input gains in the dSPACE system.

Two second-order models were fitted to the FRFs data using the frequency-domain subspace-based system identification approach, as described in [28] and [29]. The following transfer functions were found to be a good fit, as illustrated in Fig. 9,

$$
G_{c_{x} u_{x}}(s)=\frac{0.1254 s^{2}-1784 s+1.309 \times 10^{7}}{s^{2}+28.35 s+1.309 \times 10^{7}}
$$

and

$$
G_{c_{y} u_{y}}(s)=\frac{0.1006 s^{2}-1610 s+1.318 \times 10^{7}}{s^{2}+57.59 s+1.318 \times 10^{7}} .
$$

It can be inferred from transfer functions (39) and (40) that the piezoelectric tube scanner has very weakly damped resonances in $x$ and $y$ axes. In the $x$-axis, the resonance is at $576 \mathrm{~Hz}$ with a damping ratio of 0.004 and in the $y$-axis, the resonance is at $578 \mathrm{~Hz}$ with a damping ratio of 0.008 . It must be mentioned here that the nonminimum phase zeros in both transfer functions do not reflect the physical nature of the scanner, but are rather artifacts of the system identification. The subspace-based system identification approach introduces these nonminimum phase zeros in order to model delays that exist in the system due to the capacitive sensor signal processing electronics and dSPACE sampling time.

\section{Controller Design}

This section addresses design of feedback controllers undertaken in this paper. Feedback controllers for the $x$ and $y$ axes were designed independently since the scanner is treated as a two SISO systems in parallel. The key objectives of the controller design are to achieve good damping ratio for the first resonant mode of the piezoelectric tube scanner and to achieve a high closed-loop bandwidth to allow accurate tracking of the

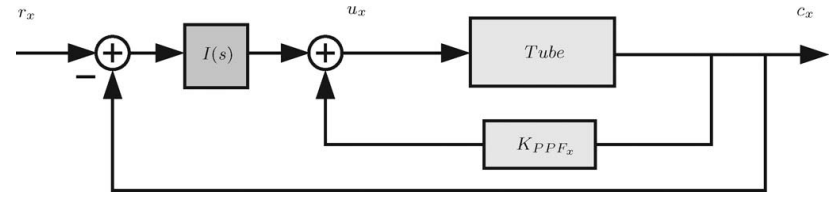

Fig. 10. Structure of the $x$-axis feedback controller. The inner feedback loop is a PPF controller designed to damp the highly resonant mode of the tube. Integral action is also incorporated to achieve satisfactory tracking.

CAV and CLV spirals. Although, the use of CAV spiral allows us to select the frequencies that will not excite the resonance of the scanner, it is still important to actively damp the scanner. External vibration and noise can result in perturbations in the AFM image if scanner's mechanical resonance is not damped. The need to damp the scanner becomes more important when it is used to track a CLV spiral input. This is because the CLV spiral input consists of high-frequency components that will inevitably excite the mechanical resonance of the scanner. Additionally, the feedback controller can minimize the effect of piezoelectric creep, which can cause further perturbation in the image [30].

Structure of the $x$-axis feedback controller is illustrated in Fig. 10. A similar controller was designed for the $y$-axis. The overall control structure consists of an inner and an outer loop. The inner loop contains a positive position feedback (PPF) controller that works to increase the overall damping of the scanner. The outer loop contains a high-gain integral controller to provide tracking. The PPF controllers were initially designed to suppress mechanical vibrations of highly resonant aerospace structures [31]. They have been successfully implemented on a range of lightly damped structures [32]-[34]. Their effectiveness in improving accuracy and bandwidth of nanopositioning systems was recently investigated in [15], and their important stability properties were established in [35]. PPF controllers have a number of important features. In particular, they have a simple structure, which makes their implementation straightforward and their transfer functions roll-off at a rate of $40 \mathrm{~dB} /$ decade at higher frequencies. The latter is important in terms of the overall effect of sensor noise on the scanner's positioning accuracy. The details of the procedure that was followed to design these PPF controllers is documented in [15]. The obtained PPF controllers can be described as follows:

$$
K_{\mathrm{PPF}_{x}}(s)=\frac{9.282 \times 10^{6}}{s^{2}+6062 s+2.736 \times 10^{7}}
$$

and

$$
K_{\mathrm{PPF}_{y}}(s)=\frac{9.313 \times 10^{6}}{s^{2}+6071 s+2.758 \times 10^{7}} .
$$

The designed control system also includes a high-gain integral controller

$$
I(s)=\frac{K_{I}}{s}
$$

as illustrated in Fig. 10. Inclusion of an integrator amounts to applying a high gain at low frequencies that reduces the effects of thermal drift, piezoelectric creep, and hysteresis to a minimum. 


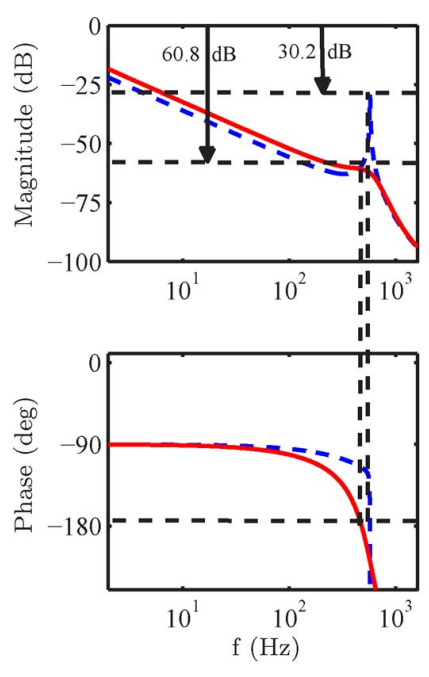

(a)

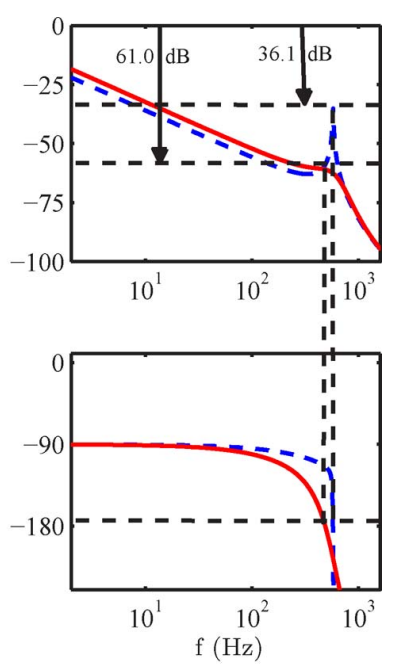

(b)
Fig. 11. Bode diagrams showing gain margins when a unity gain integral controller is cascaded with undamped (--) and damped $(-)$ scanner's transfer functions in (a) $x$ and (b) $y$ axes.

Another important benefit of the proposed combined feedback structure is the significant reduction that can be achieved in cross-coupling between various axes of the scanner.

The use of high gain in the integral controllers is made possible by the suppression of the sharp resonant peaks in the $x$ and $y$ axes due to the PPF controllers. Fig. 11 illustrates Bode diagrams showing gain margins when a unity gain integral controller is cascaded with undamped scanner's transfer functions, 1) $G_{c_{x} u_{x}}(s)$ and 2) $G_{c_{y} u_{y}}(s)$, and with damped scanner's transfer functions 3) $T_{c_{x} u_{x}}(s)$ and 4) $T_{c_{y} u_{y}}(s)$, where

$$
T_{c_{x} u_{x}}(s)=\frac{G_{c_{x} u_{x}}(s)}{1-K_{P P F_{x}}(s) G_{c_{x} u_{x}}(s)}
$$

and

$$
T_{c_{y} u_{y}}(s)=\frac{G_{c_{y} u_{y}}(s)}{1-K_{P P F_{y}}(s) G_{c_{y} u_{y}}(s)} .
$$

The gain margins for the undamped systems are 30.2 and $36.1 \mathrm{~dB}$ in $x$ and $y$ axes, respectively. This implies that the gain of the integrator $K_{I}$ is limited to less than 32 and 64 in the $x$ and $y$ axes, respectively for stability of the closed-loop systems. However, the gain margins for the damped systems are 60.8 and $61.0 \mathrm{~dB}$ in $x$ and $y$ axes, respectively. This implies that the gain of the integrator $K_{I}$ can be increased significantly from 1 to up to 1097 and 1122 in the $x$ and $y$ axes, respectively, before the closed-loop systems become unstable. In this paper, the gain of the integrators were tuned to provide high closed-loop system bandwidth, but with reasonable gain and phase margins.

\section{RESULTS}

\section{A. Tracking Performance}

The closed-loop frequency responses of the piezoelectric tube scanner were first measured using the spectrum analyzer. Fig. 12 illustrates the closed-loop frequency responses when plotted to-
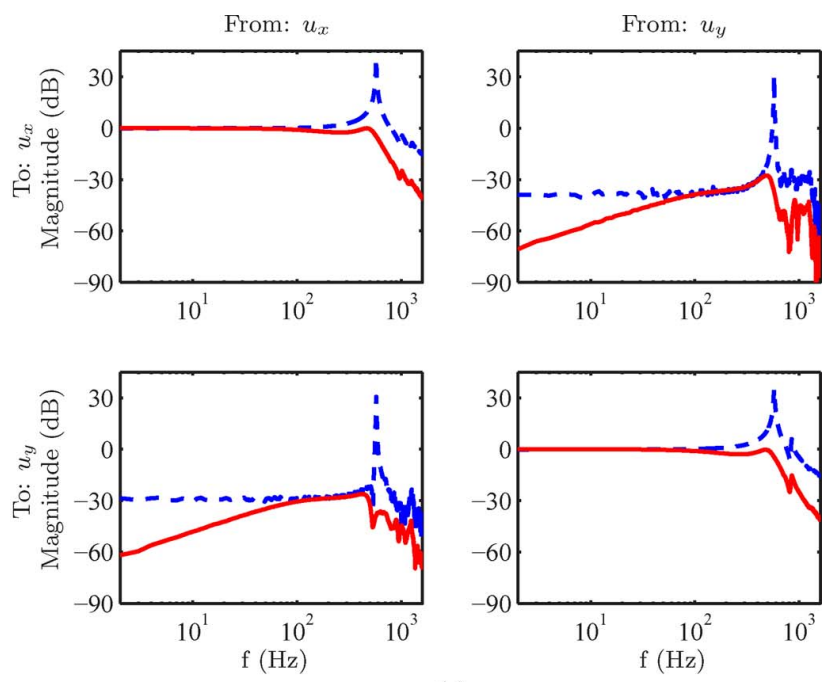

(a)
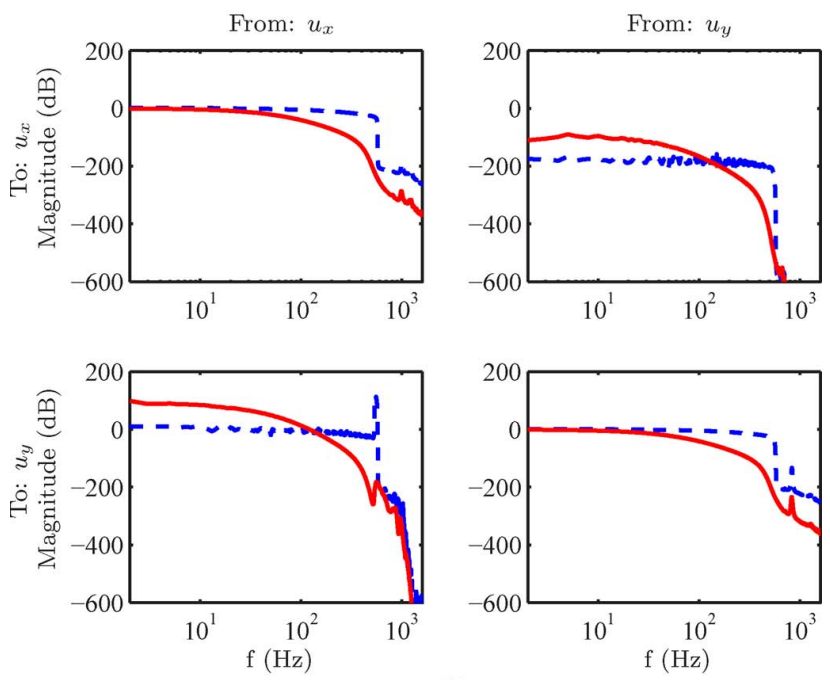

(b)

Fig. 12. Open-loop $(--)$ and closed-loop $(-)$ frequency responses of the scanner. The closed-loop system bandwidth of both axes is about $540 \mathrm{~Hz}$ and the resonant behavior of the scanner is improved by over $30 \mathrm{~dB}$ due to control action.

gether with the open-loop frequency responses of the system. By examining these FRFs, we can see that a damping of more than $30 \mathrm{~dB}$ is apparent at each resonant mode. The use of the high-gain integral controllers has resulted in a high closed-loop system bandwidth of about $540 \mathrm{~Hz}$ in both axes. However, in the closed-loop system, the frequency responses exhibit a faster phase drop rate, as compared to the open-loop system. Consequently, this results in greater phase shifts between the desired and the achieved trajectories. In this paper, the phase shifts are handled by shaping the input signals, as discussed in Section II-B.

The frequency responses for the cross-coupling of the AFM scanner in open and closed loop were also obtained and are illustrated in the off-diagonal frequency response plots of Fig. 12. In open loop, significant cross-coupling of about $30 \mathrm{~dB}$ can be observed between lateral axes of the scanner. In closed loop, the cross-coupling was substantially reduced as a result of the 

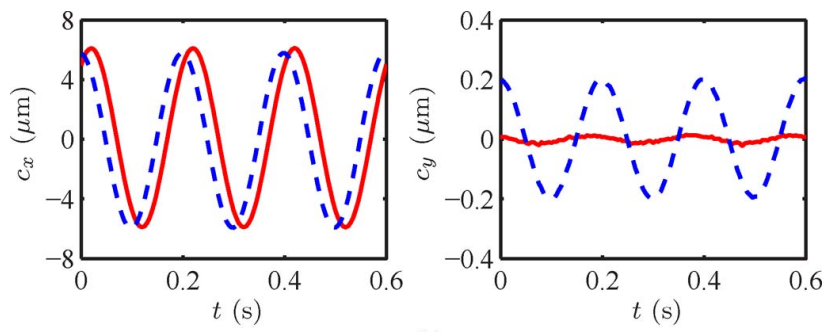

(a)
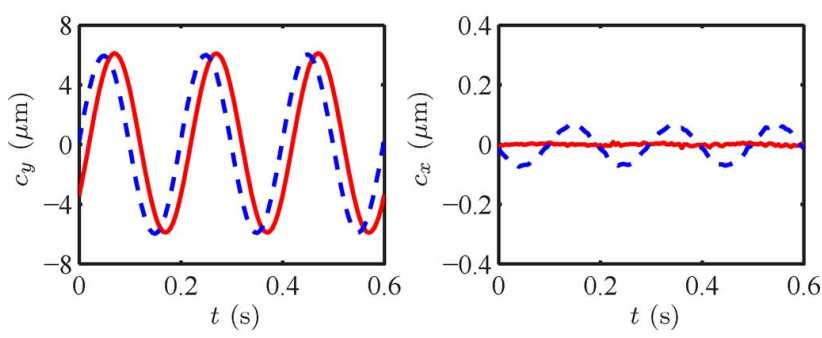

(b)

Fig. 13. Closed-loop (-) and open-loop (- - - ) tracking trajectory of (left) $5-\mathrm{Hz}$ sinusoidal signal and (right) their corresponding cross-coupling. (A small phase shift was purposely added into the close-loop time responses in order to clearly display the open- and closed-loop time responses.)

integral action. In particular, the cross-coupling is less than $50 \mathrm{~dB}$ for low frequency ranges (i.e., $\leq 8 \mathrm{~Hz}$ ). To illustrate the improvement achieved in the cross-coupling, we performed the following experiments in open and closed loops. A $5-\mathrm{Hz}$ sinusoidal signal was applied to the $+x$ electrode to produce a $12-\mu \mathrm{m}$ displacement range in the $x$-axis of the scanner. As a result of the cross-coupling from $x$ - to $y$-axis, the scanner also produced a smaller displacement range in the $y$-axis. Both displacements were then recorded and plotted in Fig. 13(a). Similar experiments were also carried out by applying the $5-\mathrm{Hz}$ sinusoidal signal to the $+y$ electrode. The subsequent displacements in the $y$ and $x$ axes were recorded and plotted in Fig. 13(b). A significant improvement can be observed by comparing the cross-coupling in open and closed loops. In closed loop, the rms cross-coupling from $x$ - to $y$-axis and from $y$ - to $x$-axis was reduced to about $6 \%$ of the cross-coupling in open loop.

The performance of the closed-loop systems were then evaluated for high-speed tracking of the CAV and CLV spirals. Both types of spiral were set up to produce spiral scans with $r_{\mathrm{end}}=6 \mu \mathrm{m}$ and number of curves $=512$, i.e., the diameter of the resulting circular image consists of 512 pixels. Fig. 14(a)-(f) illustrates tracking trajectories of the CAV spirals for $\omega_{s}=31.4$, 94.3, 188.5, 565.5, 754.0, and $1131.0 \mathrm{rad} / \mathrm{s}$. This corresponds to scanning frequencies of $f_{s}=5,15,30,90,120$, and $180 \mathrm{~Hz}$, respectively. In order to allow visual comparison of the tracking trajectories, plots in Fig. 14(a)-(f) were made to display only the trajectories between $\pm 0.15 \mu \mathrm{m}$. It can be observed that the use of the designed feedback controllers and the shaped input have resulted in excellent tracking performance of the CAV spirals up to $\omega_{s}=1131.0 \mathrm{rad} / \mathrm{s}$. In order to quantify the tracking performance, the rms tracking errors between the desired and the achieved trajectories were calculated and are tabulated in
Table I. The rms tracking error is defined as

$$
E_{\mathrm{rms}}=\sqrt{\frac{1}{t_{\text {total }}} \int_{0}^{t_{\text {total }}}\left(r(t)-r_{a}(t)\right)^{2} d t}
$$

where $r$ is the desired trajectory (or the radius) and $r_{a}=$ $\sqrt{c_{x}^{2}+c_{y}^{2}}$ is the achieved trajectory. Table I shows that $E_{\mathrm{rms}}$ increases as the spiral frequency increases. This increase is mainly due to the inability of the feedback controller to accurately track the rapid changes in the amplitude of the spiral inputs, as $\omega_{s}$ is increased. Nevertheless, at $\omega_{s}=1130.97 \mathrm{rad} / \mathrm{s}, E_{\mathrm{rms}}$ still remains relatively low, i.e., only $0.15 \%$ of the maximum scanning range (spiral's diameter).

Fig. 14(g)-(i) illustrates the tracking trajectories between $\pm 0.30 \mu \mathrm{m}$ of the CLV spirals for $v_{s}=0.2,0.6$, and $1.1 \mathrm{~mm} / \mathrm{s}$. The values of $v_{s}$ were calculated using $v_{s}=\widetilde{\omega}_{\text {end }} r_{\text {end }}$, where $\widetilde{\omega}_{\text {end }}=31.4,94.3,188.5, \mathrm{rad} / \mathrm{s}$. As mentioned earlier, the CLV spiral scans were implemented in a reversed order, i.e., from $r_{\text {end }}$ to $r_{\text {start }}$. Fig. 14(g) shows that relatively good tracking was obtained for $v_{s}=0.2 \mathrm{~mm} / \mathrm{s}$. However, for $v_{s}=0.6$ and $1.1 \mathrm{~mm} / \mathrm{s}$, Fig. 14(h) and (i) illustrates very little tracking were achieved in a small region surrounding the center of the spirals, where the frequency components of the input signals have increased well beyond the bandwidth of the closed-loop system. Nonetheless, Table I shows that the $E_{\text {rms }}$ for the CLV spirals is still relatively small since most of the tracking errors were limited only to the center of the resulting spiral scan.

\section{B. AFM Imaging}

Having analyzed the performance of the closed-loop system in tracking the CAV and CLV spirals, we then moved on to investigate the use of spiral scanning in generating AFM images. The spiral scans were set up to produce images with $r_{\text {end }}=$ $6.5 \mu \mathrm{m}$ and number of curves $=512$, i.e., the diameter of the resulting circular image consists of 512 pixels. However, before performing the spiral scans, the rms of mapping errors $E_{\mathrm{map} \text { rms }}$ for the CAV and CLV spirals scans at different angular and linear velocities are calculated and tabulated in Table II. Note that different sampling frequencies $f_{\text {samp }}$ were used in order to minimize the computing time for searching the nearest spiral point corresponding the each raster point. Additionally, the sampling frequency is also limited by computational power of the dSPACE rapid prototyping system. Table II shows that the $E_{\mathrm{map}_{\mathrm{rms}}}$ are very small and less then the pitch of the spiral trajectory and the raster points, i.e., $25.44 \mathrm{~nm}$. Thus, they can be ignored.

A calibration grating NT-MDT TGQ1 with a $20-\mathrm{nm}$ feature height and a 3- $\mu \mathrm{m}$ period was used as an imaging sample. The AFM was set up to scan the sample in constant-height contact mode using a contact AFM probe with a nominal spring constant of $0.2 \mathrm{~N} / \mathrm{m}$ and resonance frequency of $13 \mathrm{kHz}$. The constant-height contact mode was used here as the commercial AFM controller that controls the vertical positioning of the scanner is not fast enough to track the sample topography for high-speed scans. During each scan, the AFM probe is deflected due to its interactions with the sample. The probe 


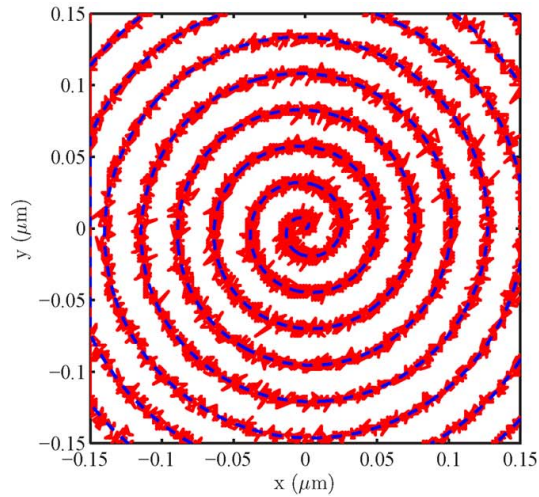

(a)

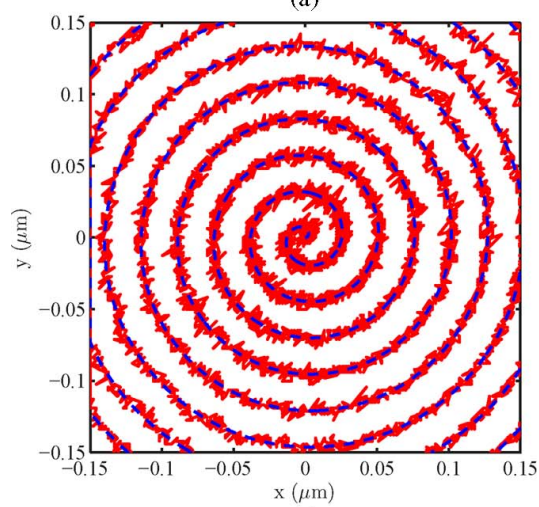

(b)

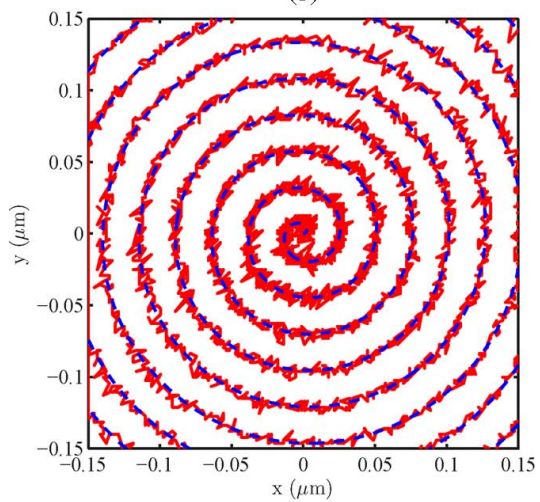

(c)

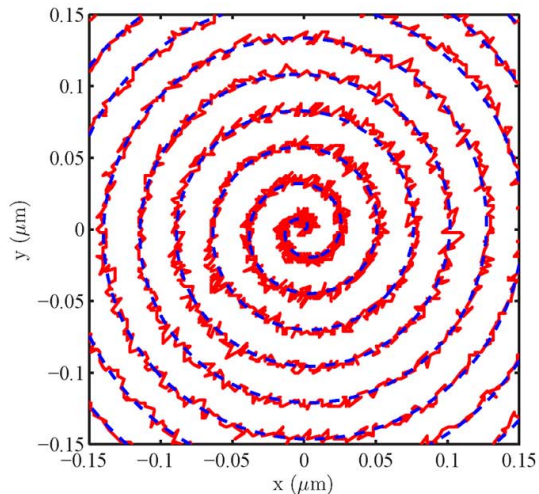

(d)

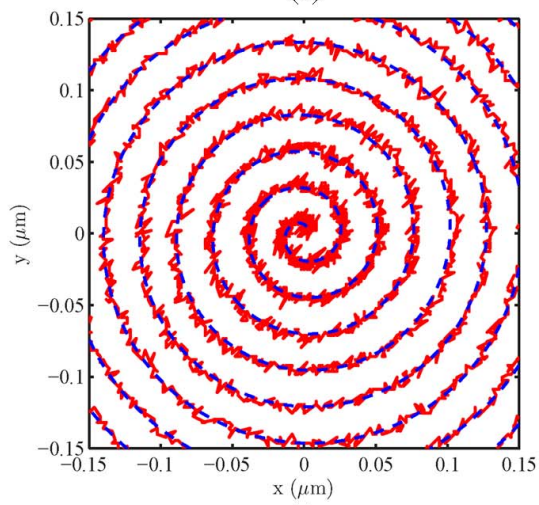

(e)

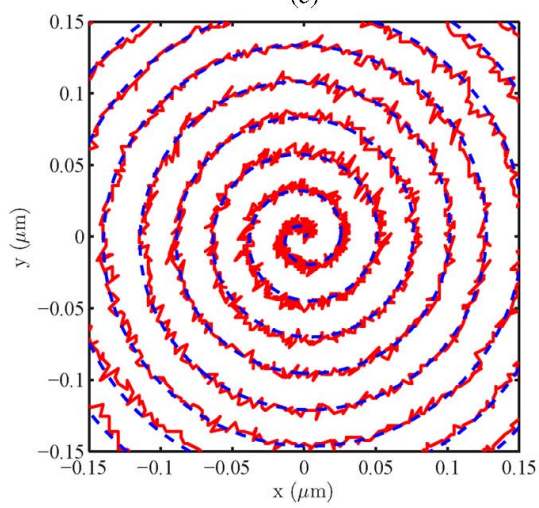

(f)

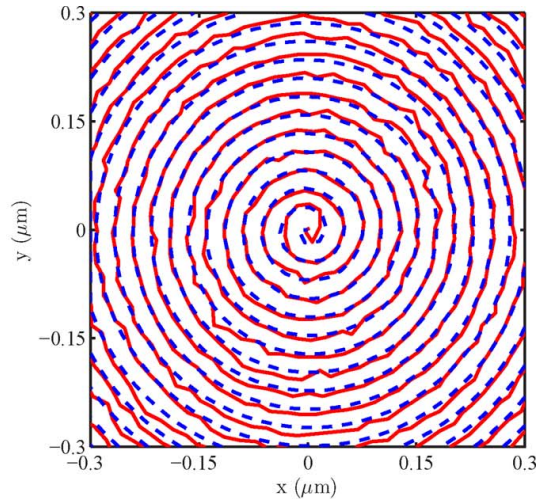

(g)

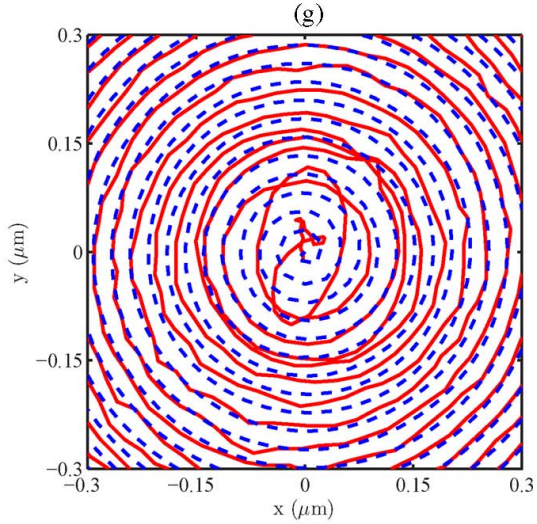

(h)

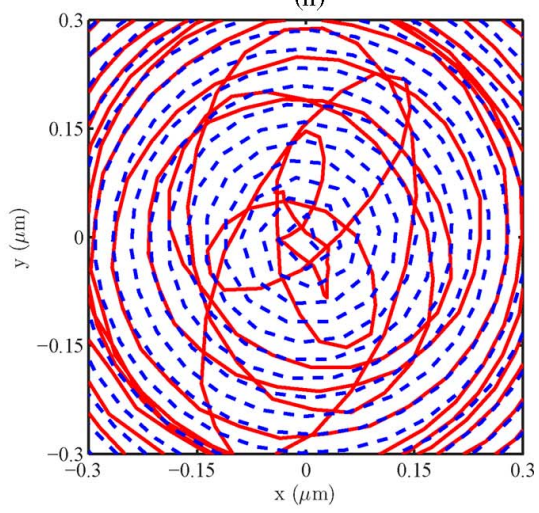

(i)

Fig. 14. First two columns: (a)-(f) Tracking trajectories of CAV spirals between between $\pm 0.15 \mu \mathrm{m}$ in closed loop for $\omega_{s}=31.4,94.3$, 188.5, 565.5, 754.0, and $1131.0 \mathrm{rad} / \mathrm{s}$. Third column: (g)-(i) Tracking trajectories of CLV spirals between $\pm 0.30 \mu \mathrm{m}$ in closed loop for $v_{s}=0.2,0.6$, and $1.1 \mathrm{~mm} / \mathrm{s}$. The pitch of the spirals was set at $0.0235 \mu \mathrm{m}$. Solid line is the achieved response and dashed line is the desired trajectory.

TABLE I

RMS VALUES OF TRACKING ERROR AND TOTAL SCANNING TIME FOR CAV AND CLV SPIRAL SCANS. THE NUMBER OF CURVE FOR THESE SPIRAL SCANS WAS SET TO 512 .

\begin{tabular}{|c|c|c||c|c|c|}
\hline \multicolumn{3}{|c||}{ CAV Spiral } & \multicolumn{3}{c|}{ CLV Spiral } \\
\hline $\begin{array}{c}\omega_{s} \\
\text { (radians/s) }\end{array}$ & $\begin{array}{c}E_{\text {rms }} \\
(\mathrm{nm})\end{array}$ & $\begin{array}{c}t_{\text {total }} \\
(\mathrm{s})\end{array}$ & $\begin{array}{c}v_{s} \\
(\mathrm{~mm} / \mathrm{s})\end{array}$ & $\begin{array}{c}E_{\text {rms }} \\
(\mathrm{nm})\end{array}$ & $\begin{array}{c}\widetilde{t}_{\text {total }} \\
(\mathrm{s})\end{array}$ \\
\hline 31.4 & 2.81 & 51.10 & 0.19 & 3.60 & 25.55 \\
94.3 & 4.60 & 17.03 & 0.57 & 7.26 & 8.52 \\
188.5 & 5.19 & 8.52 & 1.13 & 10.67 & 4.26 \\
565.5 & 10.38 & 2.84 & - & - & - \\
754.0 & 11.30 & 2.13 & - & - & - \\
1131.0 & 18.16 & 1.42 & - & - & - \\
\hline
\end{tabular}

deflection was measured and later used to construct AFM images of the sample topography. Fig. 15(a)-(f) illustrates AFM images generated using the CAV spiral scans with $\omega_{s}=31.4$,
TABLE II

RMS VALUeS of SPIRAL to RASTER POINTS MAPPING ERROR FOR CAV AND CLV SPIRAL SCANS

\begin{tabular}{|c|c|c||c|c|c|}
\hline \multicolumn{3}{|c||}{ CAV Spiral } & \multicolumn{3}{c|}{ CLV Spiral } \\
\hline $\begin{array}{c}\omega_{s} \\
(\text { radians/s) }\end{array}$ & $\begin{array}{c}f_{\text {samp }} \\
(\mathrm{kHz})\end{array}$ & $\begin{array}{c}E_{\text {map }_{R M S}} \\
(\mathrm{~nm})\end{array}$ & $\begin{array}{c}v_{s} \\
(\mathrm{~mm} / \mathrm{s})\end{array}$ & $\begin{array}{c}f_{\text {samp }} \\
(\mathrm{kHz})\end{array}$ & $\begin{array}{c}E_{\text {map }}(\mathrm{nMS}) \\
(\mathrm{nm})\end{array}$ \\
\hline 31.4 & 10 & 2.49 & 0.19 & 10 & 2.61 \\
94.3 & 20 & 2.64 & 0.57 & 20 & 2.83 \\
188.5 & 20 & 3.12 & 1.13 & 20 & 3.49 \\
565.5 & 40 & 3.42 & - & - & - \\
754.0 & 60 & 3.51 & - & - & - \\
1131.0 & 60 & 3.75 & - & - & - \\
\hline
\end{tabular}

$94.3,188.5,565.5,754.0$, and $1131.0 \mathrm{rad} / \mathrm{s}$. It can be observed from these figures that the obtained images are of a good quality and the profile of the calibration grating is well captured. In 


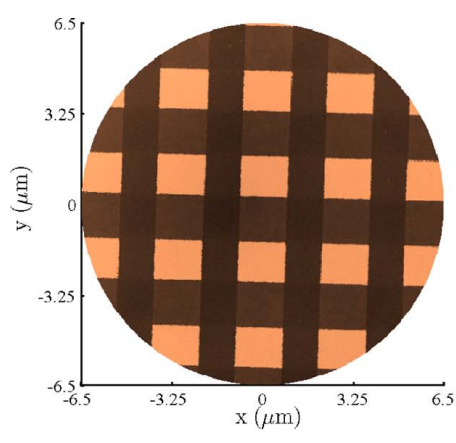

(a)

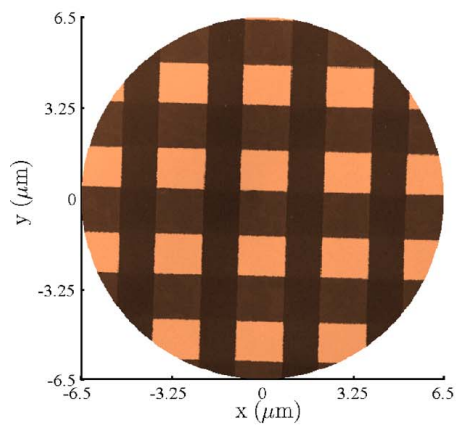

(b)

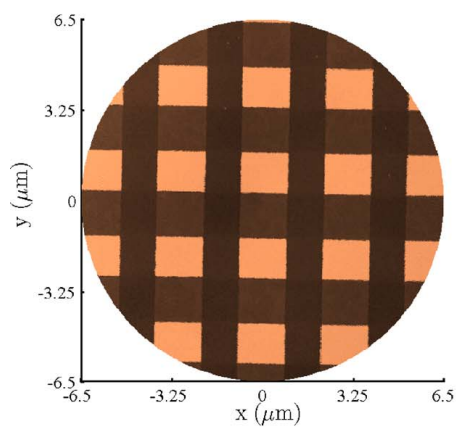

(c)

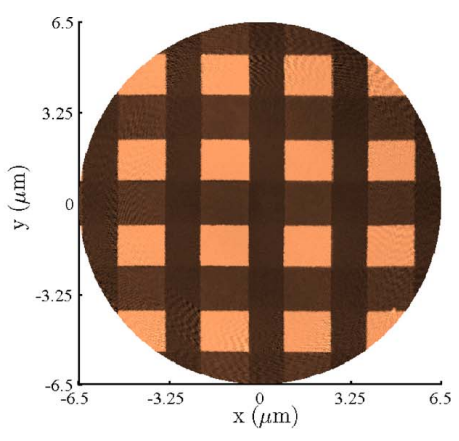

(d)

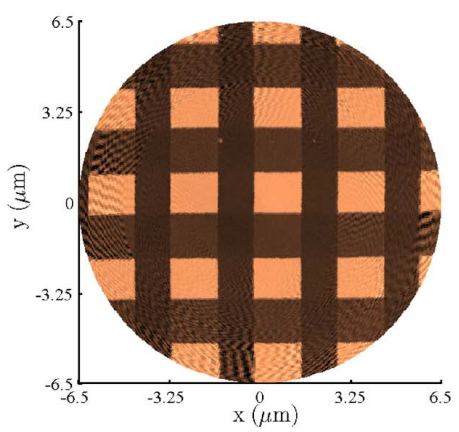

(e)

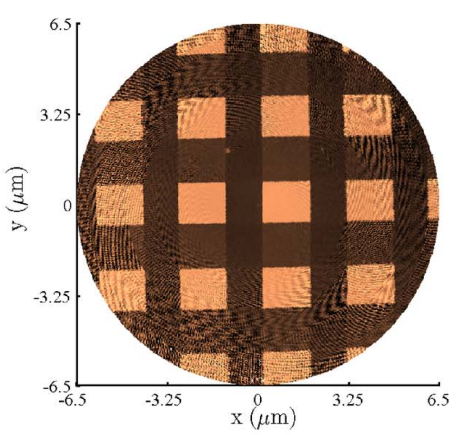

(f)

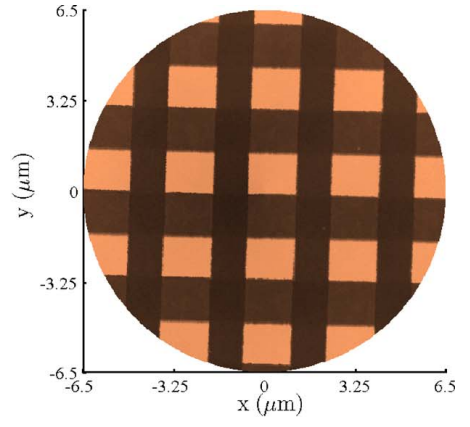

(g)

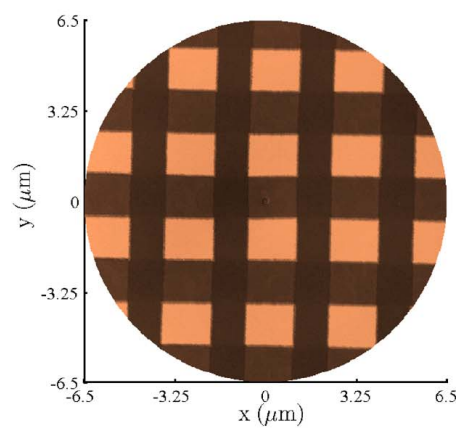

(h)

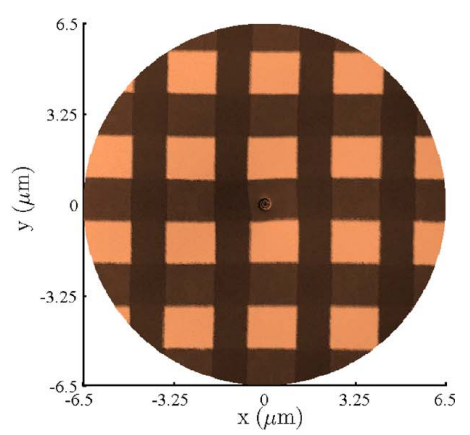

(i)

Fig. 15. AFM images of NT-MDT TGQ1 grating scanned in closed loop using the CAV spiral scanning mode for (a)-(f) $f_{s}=5,15,30,90,120$, and 180 Hz (which corresponds to $\omega_{s}=31.4,94.3,188.5,565.5,754.0$, and $1131.0 \mathrm{rad} / \mathrm{s}$ ) and using the CLV spiral scanning mode for $(\mathrm{g})-(\mathrm{i}) v_{s}=0.2,0.6$, and $1.1 \mathrm{~mm} / \mathrm{s}$. The number of curve for these AFM images was set to 512 .

particular, the images are free from typical distortions caused by tracking errors, scanner vibrations, hysteresis, and creep. It is also worth mentioning that the areas around the outer edges of the images was also relatively well imaged. However, during high-speed scans with $\omega_{s}=565.5 \mathrm{rad} / \mathrm{s}$ and above, a wavelike artifact can be observed around the outer edge of the AFM images. Upon a closer examination of the probe deflection signals, we found that the artifacts were a result of the excitation of the probe's resonance. Fig. 16 illustrates the probe deflection signals between $r=0.597$ and $0.6 \mu \mathrm{m}$ for $\omega_{s}=31.4$ and $1131.0 \mathrm{rad} / \mathrm{s}$. Fig. 16(a) shows that during a low-speed scan the probe deflection signal is free of vibrations. However at a high-speed scan, Fig. 16(b) shows that the probe vibrates at its resonance frequency $(\approx 12 \mathrm{kHz})$ after every step change in the sample profile. Thus, the image quality can be improved by using a stiffer microcantilever. This would allow for much higher scan frequencies, approaching the mechanical resonance of the scanner.

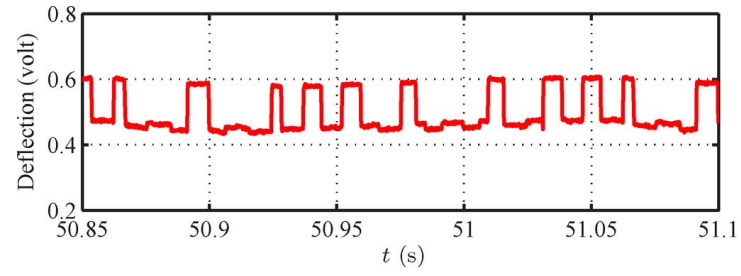

(a)

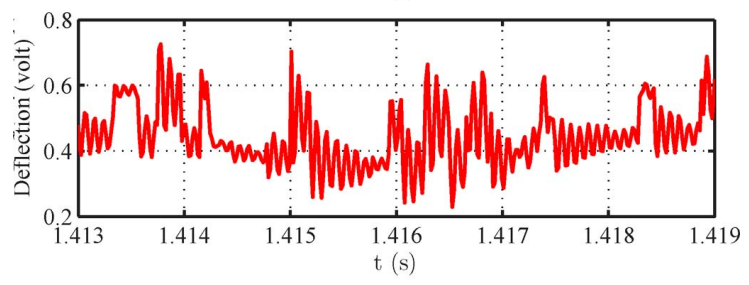

(b)

Fig. 16. Probe deflection signals showing the profile of the calibration grating for (a) $\omega_{s}=31.4 \mathrm{rad} / \mathrm{s}$ and (b) $\omega_{s}=754.0 \mathrm{rad} / \mathrm{s}$. 


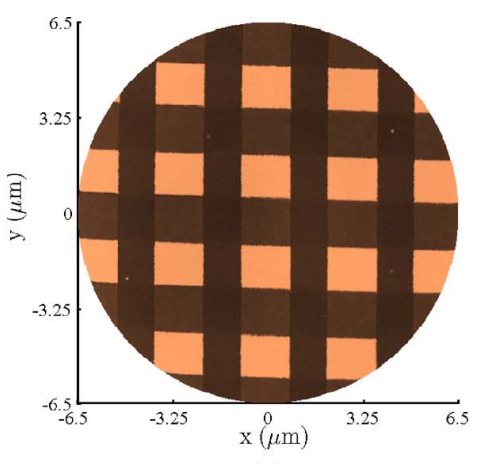

(a)

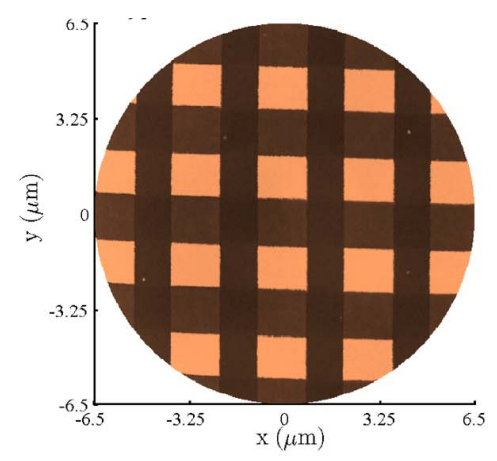

(b)

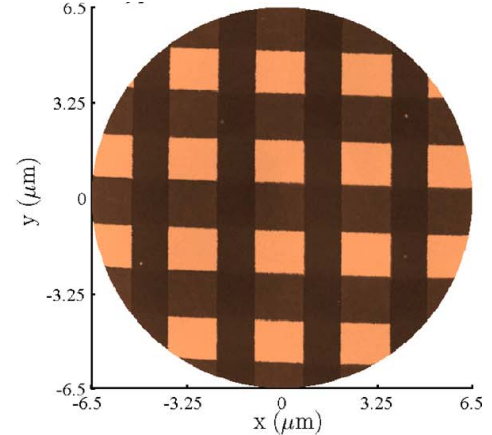

(c)

Fig. 17. AFM images of NT-MDT TGQ1 grating scanned in open loop using the CAV spiral for (a) $-(\mathrm{c}) f_{s}=5,30$, and $90 \mathrm{~Hz}$. The number of curve for these AFM images was set to 512 .

Next, a similar AFM setting was used to generate similar images using the CLV spiral scanning mode. Fig. 15(g)-(i) illustrates the generated AFM images for $v_{s}=0.2,0.6$, and $1.1 \mathrm{~mm} / \mathrm{s}$. For $v_{s}=0.2 \mathrm{~mm} / \mathrm{s}$, it can be observed that the profile of the calibration grating was well imaged. This is in agreement with the good tracking performance achieved at this scanning speed, as illustrated in Fig. 14(g). However, for higher values of $v_{s}$, Fig. 15(h) and (i) illustrates that a small hole-like artifact is formed at the center of each image. This is due to the loss of tracking control when the frequency components of the input signals have increased well beyond the bandwidth of the closedloop system. The lack of tracking control has also resulted in a slightly skewed AFM image around the center of the spiral for $v_{s}=1.1 \mathrm{~mm} / \mathrm{s}$.

Finally, we would like to evaluate the capability of the CAV spiral scans in generating the AFM images when operated in open loop . The use of single-frequency input, as mentioned in Section II-A, would allow the open-loop tracking of the CAV spirals to be performed rather accurately. However, to achieve this, one has to deal with the nonlinearities of the piezoelectric tube scanner, particularly with hysteresis and creep. In this paper, the effect of hysteresis was significantly reduced by the use of the charge amplifiers instead of voltage amplifiers to drive both axes of the scanner. As for the creep, its effect was minimized by simply waiting for a considerable amount of time for it to disappear before performing the scans. Fig. 17(a)-(c) illustrates AFM images generated using the CAV spiral scans operated in open loop for $f_{s}=5,30$, and $90 \mathrm{~Hz}$. It can be observed from these images that the spiral scanning methods works surprisingly well when operated in an open loop. This could be partially due to the fact that by controlling charge, we have managed to substantially minimize the effect of hysteresis. However, even if the scanner were driven with voltage amplifiers, the hysteresis nonlinearity could have been compensated for by perturbing the input signal. This would be rather straightforward due to the single-tone nature of signals applied to the $x$ and $y$ electrodes of the piezoelectric tube scanner.

\section{CONCLUSIONS AND Future Work}

In conclusion, in this paper, we demonstrated how a CAV and CLV spiral scans can be used to obtain AFM images. It is possible to achieve high-speed atomic force microscopy using the CAV spiral scanning, but other issues like the vibrations in the AFM probe need to be considered and addressed. The use of CLV-mode spiral scanning requires a high-bandwidth controller for accurate tracking of the input signals. Apart from the aforementioned artifacts formed at the center of the CLV spiral, the obtained AFM images have good qualities. We also demonstrated that the proposed method could work well without using a feedback controller around the AFM scanner. The possibility of using spiral scanning in other SPM applications such as STM should also be explored in the future.

\section{REFERENCES}

[1] G. Binnig, C. F. Quate, and C. Gerber, "Atomic force microscope," Phys. Rev. Lett., vol. 56, no. 9, pp. 930-933, Mar. 1986.

[2] E. Meyer, H. J. Hug, and R. Bennewitz, Scanning Probe Microscopy. Berlin, Germany: Springer-Verlag, 2004.

[3] B. Bhushan, Springer Handbook of Nanotechnology. Berlin, Germany: Springer-Verlag, 2006.

[4] S. O. R. Moheimani, "Invited review article: Accurate and fast nanopositioning with piezoelectric tube scanners: Emerging trends and future challenges," Rev. Sci. Instrum., vol. 79, no. 7, pp. 071101-1-071101-11, Jul. 2008.

[5] Y. K. Yong, S. Aphale, and S. O. R. Moheimani, "Design, identification, and control of a flexure-based xy stage for fast nanoscale positioning," IEEE Trans. Nanotechnol., vol. 8, no. 1, pp. 46-54, Jan. 2009.

[6] G. Schitter, K. Astrom, B. DeMartini, P. Thurner, K. Turner, and P. Hansma, "Design and modeling of a high-speed afm-scanner," IEEE Trans. Control Syst. Technol., vol. 15, no. 5, pp. 906-915, Sep. 2007.

[7] B. P. Lathi, Linear Systems and Signals, 2nd ed. London, U.K.: Oxford Univ. Press, 2004.

[8] D. Croft, G. Shed, and S. Devasia, "Creep, hysteresis, and vibration compensation for piezoactuators: Atomic force microscopy application," $J$. Dyn. Sys. Meas. Control, vol. 123, no. 1, pp. 35-43, Mar. 2001.

[9] S. Devasia, E. Eleftheriou, and S. Moheimani, "A survey of control issues in nanopositioning," IEEE Trans. Control Syst. Technol., vol. 15, no. 5, pp. 802-823, Sep. 2007.

[10] N. Tamer and M. Dahleh, "Feedback control of piezoelectric tube scanners," in Proc. 33rd IEEE Conf. Decision Control, Dec. 1994, vol. 2, pp. $1826-1831$.

[11] A. Daniele, S. Salapaka, M. Salapaka, and M. Dahleh, "Piezoelectric scanners for atomic force microscopes: Design of lateral sensors, identification, and control," in Proc. Amer. Control Conf., 1999, vol. 1, pp. 253-257.

[12] K. Leang and S. Devasia, "Feedback-linearized inverse feedforward for creep, hysteresis, and vibration compensation in AFM piezoactuators," IEEE Trans. Control Syst. Technol., vol. 15, no. 5, pp. 927-935, Sep. 2007.

[13] S. Salapaka, A. Sebastian, J. P. Cleveland, and M. V. Salapaka, "High bandwidth nano-positioner: A robust control approach," Rev. Sci. Instrum., vol. 73, no. 9, pp. 3232-3241, Sep. 2002. 
[14] G. Schitter and A. Stemmer, "Identification and open-loop tracking control of a piezoelectric tube scanner for high-speed scanning-probe microscopy," IEEE Trans. Control Syst. Technol., vol. 12, no. 3, pp. 449-454, May 2004.

[15] B. Bhikkaji, M. Ratnam, A. Fleming, and S. Moheimani, "Highperformance control of piezoelectric tube scanners," IEEE Trans. Control Syst. Technol., vol. 15, no. 5, pp. 853-866, Sep. 2007.

[16] W. Zhang, L. Miao, Y. Zheng, Z. Dong, and N. Xi, "Feedback control implementation for AFM contact-mode scanner," in Proc. 3rd IEEE Int. Conf. Nano/Micro Eng. Mol. Syst. (NEMS), Jan. 2008, pp. 617-621.

[17] C. Lee and S. M. Salapaka, "Robust broadband nanopositioning: Fundamental trade offs, analysis, and design in a two-degree-of-freedom control framework," Nanotechnology, vol. 20, no. 3, pp. 035501-1-035501-16, 2009.

[18] A. N. Labinsky, G. A. J. Reynolds, and J. Halliday, "A disk recording system and a method of controlling the rotation of a turn table in such a disk recording system," WO Patent 93/13524, Jul. 1993.

[19] A. D. L. Humphris, J. K. Hobbs, and M. J. Miles, "Ultrahigh-speed scanning near-field optical microscopy capable of over 100 frames per second," Appl. Phys. Lett., vol. 83, no. 1, pp. 6-8, 2003.

[20] G. Schitter and M. J. Rost, "Scanning probe microscopy at video-rate," Mater. Today, vol. 11, no. 1, pp. 40-48, 2008.

[21] T. Ando, T. Uchihashi, and T. Fukuma, "High-speed atomic force microscopy for nano-visualization of dynamic biomolecular processes," Progr. Surface Sci., vol. 83, no. 7-9, pp. 337-437, Nov. 2008.

[22] L. M. Picco, L. Bozec, A. Ulcinas, A. J. Engledew, M. Antognozzi, M. A. Horton, and M. J. Miles, "Breaking the speed limit with atomic force microscopy," Nanotechnology, vol. 18, no. 4, pp. 044030-1-044030-4, 2007.

[23] H. Yamashita, K. Voitchovsky, T. Uchihashi, S. A. Contera, J. F. Ryan, and T. Ando, "Dynamics of bacteriorhodopsin 2-D crystal observed by high-speed atomic force microscopy," J. Struct. Biol., vol. 167, no. 2, pp. 153-158, 2009.

[24] J. W. Rutter, Geometry of Curves. Boca Raton, FL: Chapman and Hall/CRC, 2000.

[25] A. J. Fleming and S. O. R. Moheimani, "Sensorless vibration suppression and scan compensation for piezoelectric tube nanopositioners," IEEE Trans. Control Syst. Technol., vol. 14, no. 1, pp. 33-44, Jan. 2006.

[26] A. Fleming and K. Leang, "Charge drives for scanning probe microscope positioning stages," Ultramicroscopy, vol. 108, no. 12, pp. 1551-1557, 2008.

[27] R. Pintelon and J. Schoukens, System Identification: A Frequency Domain Approach. New York: IEEE Press, 2001.

[28] T. McKelvey, H. Akcay, and L. Ljung, "Subspace-based multivariable system identification from frequency response data," IEEE Trans. Autom. Control, vol. 41, no. 7, pp. 960-979, Jul. 1996.

[29] T. McKelvey, A. Fleming, and S. O. R. Moheimani, "Subspace-based system identification for an acoustic enclosure," J. Vibration Acoust., vol. 124 , no. 3, pp. 414-419, 2002.

[30] S. O. R. Moheimani and A. J. Fleming, Piezoelectric Transducers for Vibration Control and Damping. Germany: Springer-Verlag, 2006.

[31] J. L. Fanson and T. K. Caughey, "Positive position feedback-control for large space structures," AIAA J., vol. 28, no. 4, pp. 717-724, Apr. 1990.

[32] K. H. Rew, J. H. Han, and I. Lee, "Multi-modal vibration control using adaptive positive position feedback," J. Intell. Mater. Syst. Struct., vol. 13, no. 1, pp. 13-22, Jan. 2002.

[33] G. Song, S. P. Schmidt, and B. N. Agrawal, "Experimental robustness study of positive position feedback control for active vibration suppression,” J. Guid., Control Dyn., vol. 25, no. 1, pp. 179-182, 2002.

[34] S. Moheimani, B. Vautier, and B. Bhikkaji, "Experimental implementation of extended multivariable ppf control on an active structure," IEEE Trans. Control Syst. Technol., vol. 14, no. 3, pp. 443-455, May 2006.

[35] A. Lanzon and I. Petersen, "Stability robustness of a feedback interconnection of systems with negative imaginary frequency response," IEEE Trans. Autom. Control, vol. 53, no. 4, pp. 1042-1046, May 2008.

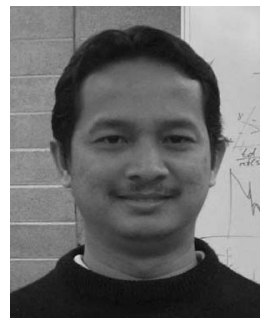

Iskandar A. Mahmood was born in Malaysia, in 1975. He received the B.Eng. and M.Sc. degrees in mechatronics engineering from the International Islamic University Malaysia, Kuala Lumpur, Malaysia, in 1999 and 2004, respectively. He is currently working toward the Ph.D. degree in electrical engineering with the University of Newcastle, Callaghan, N.S.W., Australia.

Since 2005, he has been with the Laboratory of Dynamics and Control of Nanosystems, University of Newcastle.

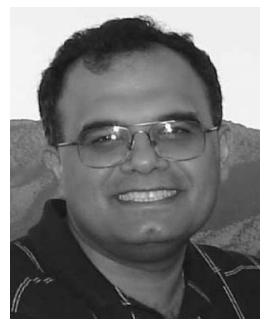

S. O. Reza Moheimani (SM'00) received the Ph.D. degree in electrical engineering from the University of New South Wales, Australian Defence Force Academy, Canberra, A.C.T., Australia, in 1996.

He is currently a Professor with the School of Electrical Engineering and Computer Science, University of Newcastle, Callaghan, N.S.W., Australia, where he holds an Australian Research Council Future Fellowship. He is an Associate Director of the Australian Research Council Centre for Complex Dynamic Systems and Control, an Australian Government Centre of Excellence. He has held several visiting appointments at IBM Zurich Research Laboratory, Switzerland. He has authored or coauthored two books, several edited volumes, and more than 200 refereed articles in archival journals and conference proceedings. His research interests include applications of control and estimation in nanoscale positioning systems for scanning probe microscopy, and control of electrostatic microactuators in microelectromechanical systems and data storage systems.

Prof. Moheimani is a Fellow of the Institute of Physics, U.K. He is a recipient of the 2007 IEEE TRANSACTIONS ON CONTROL SYSTEMS TECHNOLOGY Outstanding Paper Award and a recipient of the 2009 IEEE CSS Control Systems Technology Award. He has served on the Editorial Board of a number of journals including the IEEE TRANSACTIONS ON CONTROL SYSTEMS TECHNOLOGY, and chaired several international conferences and workshops.

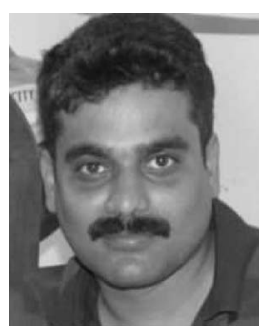

Bharath Bhikkaji received the Bachelor's degree in mathematics from the University of Madras, Chennai, India, in 1994, the Master's degree in engineering from the Department of Electrical Communication Engineering, Indian Institute of Science, Bengaluru, India, in 1998, and the Ph.D. degree in signal processing from Uppsala University, Uppsala, Sweden, in 2004.

During 2004-2009, he was as a Research Associate with University of Newcastle, Callaghan, Australia. He is currently an Assistant Professor with the Department of Electrical Engineering, Indian Institute of Technology Madras, Chennai. His research interests include applications of system identification, signal processing, and control systems design to vibration damping in flexible structures. 